\title{
Hilbert Schemes as Moduli of Higgs Bundles and Local Systems
}

\section{Michael Groechenig ${ }^{1,2}$}

\author{
${ }^{1}$ Mathematical Institute, 24-29 St Giles', Oxford OX1 3LB, UK and \\ ${ }^{2}$ Section de mathématiques, École Polytechnique Fédérale de Lausanne, \\ CH-1015 Lausanne, Switzerland
}

Correspondence to be sent to: groechenig@maths.ox.ac.uk

We construct five families of 2D moduli spaces of parabolic Higgs bundles (respectively, local systems) by taking the equivariant Hilbert scheme of a certain finite group acting on the cotangent bundle of an elliptic curve (respectively, twisted cotangent bundle). We show that the Hilbert scheme of $m$ points of these surfaces is again a moduli space of parabolic Higgs bundles (respectively, local systems), confirming a conjecture of Boalch in these cases and extending a result of Gorsky-Nekrasov-Rubtsov. Using the McKay correspondence, we establish the autoduality conjecture for the derived categories of the moduli spaces of Higgs bundles under consideration.

\section{Introduction}

Let $X$ be a smooth projective curve defined over an algebraically closed field $k$. A Higgs bundle on $X$ is a pair consisting of a vector bundle $E$ on $X$ and a Higgs field $\theta$ given by a morphism of locally free sheaves

$$
\theta: E \rightarrow E \otimes \Omega_{X}^{1}
$$

where $\Omega_{X}^{1}$ denotes the sheaf of 1 -forms on $X$. The study of Higgs bundles on curves has been initiated by Hitchin [28] in relation to the construction of solutions to a dimensional reduction of the Yang-Mills equation. An essential device to study Higgs bundles has been the Hitchin map, which associates to a pair $(E, \theta)$, the characteristic polynomial 
of the Higgs field $\theta$. The zero scheme cut out by the characteristic polynomial in $T^{*} X$ is a possibly highly singular curve, referred to as the spectral curve. Passing to moduli spaces and fixing the rank $n$ of the vector bundle $E$ reveals a beautiful geometry. The moduli space of Higgs bundles $\mathcal{M}$ fibers over an affine space $\mathcal{A}$, giving rise to an integrable system [27]

$$
\chi: \mathcal{M} \rightarrow \mathcal{A} .
$$

The fibers of the Hitchin map can be understood as compactified Picard varieties of the spectral curves. While this implies that the generic Hitchin fiber is an abelian variety, the global geometry of the Hitchin fibration remains mysterious.

Here, we describe five infinite families of examples of moduli spaces of parabolic Higgs bundles (see Definition 2.10), whose geometry is nontrivial but remains manageable. Every family is constructed by taking the Hilbert scheme of length $m$ points of certain surfaces arising as moduli spaces of parabolic Higgs bundles.

The 2D examples of moduli spaces of parabolic Higgs bundles, which we will consider, are in correspondence with the affine Dynkin diagrams $\tilde{A}_{0}, \tilde{D}_{4}, \tilde{E}_{6}, \tilde{E}_{7}$, and $\tilde{E}_{8}$ and are constructed as certain resolutions of singularities of quotients of cotangent bundles of elliptic curves.

In the simplest case of $\tilde{A}_{0}$, the corresponding moduli space in this list is the cotangent bundle of an elliptic curve $E$. For $\tilde{D}_{4}$, one considers the natural action of the group $\Gamma=\mathbb{Z} / 2 \mathbb{Z}$ on $T^{*} E$ given by the inverse morphism $x \mapsto-x$ of $E$. The 2D moduli spaces of this type are certain crepant resolutions of the GIT quotients

$$
T^{*} E / \Gamma,
$$

given by the $\Gamma$-Hilbert schemes of $T^{*} E$. The corresponding moduli problem of parabolic Higgs bundles is given by rank 2 and orbifold degree 0 parabolic Higgs bundles on $\mathbb{P}^{1}$, where the Higgs field $\theta$ is allowed to acquire order one poles at four marked points, such that the residue of $\theta$ is nilpotent with respect to the flags at these given points. We point out that $\mathbb{P}^{1}$ is the scheme-theoretic quotient of $E$ with respect to the above action. The four marked points correspond to the four fixed points of this action, that is, the places of ramification of the quotient map $E \rightarrow E / \Gamma$. The data describing this moduli problem of parabolic bundles is encoded in the diagram below:

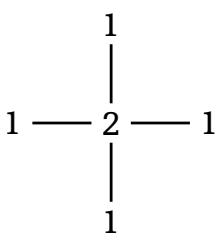


As the reader will have already guessed, the number at the central vertex indicates the rank of the parabolic bundle, and every tail corresponds to a marked points, and the numbers at the remaining vertices indicate the ranks of the flags given by the parabolic structure.

In the $\tilde{E}_{7}$-case, we pick an elliptic curve $E$ with an automorphism of order 4 . This is an elliptic curve with a special form of complex multiplication, which corresponds to the lattice of Gaussian integers $\mathbb{Z}[i] \subset \mathbb{C}$. According to [50, p. 483], this elliptic curve corresponds to the equation

$$
y^{2}=x^{3}+x
$$

and the $\mathbb{Z} / 4 \mathbb{Z}$-action is generated by $(x, y) \mapsto(-x, i y)$. Taking the scheme-theoretic quotient of $E$ as in the example above, we obtain $\mathbb{P}^{1}$ with three marked points. And it turns out that the $\Gamma$-Hilbert scheme of $T^{*} E$ is a moduli space for rank 4 orbifold degree 0 parabolic Higgs bundles on this marked rational curve. The Higgs field is again allowed to acquire order 1 singularities at the marked points, as long as the residues are nilpotent with respect to the parabolic structure encoded in the diagram:

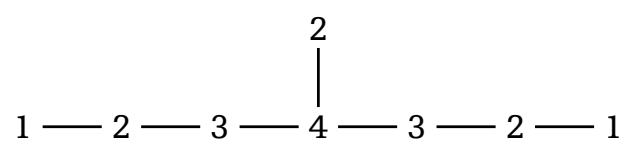

For the graphs $\tilde{E}_{6}$ and $\tilde{E}_{8}$, we proceed analogously with an elliptic curve $E$ with a nontrivial $\mathbb{Z} / 3 \mathbb{Z}$ - and $\mathbb{Z} / 6 \mathbb{Z}$-action, respectively. Over the field of complex numbers, such a curve is given by the lattice of Eisenstein integers $\mathbb{Z}[\omega] \subset \mathbb{C}$, where $\omega$ is a primitive third root of unity. According to [50, p. 483], an explicit equation for this curve is given by

$$
y^{2}+y=x^{3}
$$

The $\mathbb{Z} / 6 \mathbb{Z}$-action is generated by $\mathbb{Z} / 3 \mathbb{Z}$-action given by $\xi \cdot(x, y)=(\xi x, y)$ for every third root of unity $\xi$, and the $\mathbb{Z} / 2 \mathbb{Z}$-action induced by the inverse map of $E$. The corresponding parabolic bundles are of rank 3, respectively, rank 6 and orbifold degree 0 . The respective parabolic structure is encoded in the diagrams:

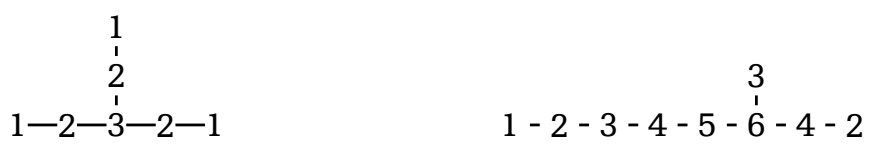


The following theorem will be proved as Theorem 4.1 .

Theorem 1.1. Let $E$ be an elliptic curve with an action of a finite group $\Gamma$ as described above. Then there is an isomorphism of the $\Gamma$-Hilbert scheme of $T^{*} E$ with a 2D moduli space of stable parabolic Higgs bundles. In particular, these moduli spaces are crepant resolutions of the GIT quotients $T^{*} E / \Gamma$.

Our main result is that the Hilbert scheme of length $m$ points of each of these surfaces is equivalent to a moduli space of parabolic Higgs bundles.

Theorem 1.2. Let $\mathcal{M}$ be one of the smooth moduli space of stable parabolic Higgs bundles of dimension 2, with stability conditions, degree, and dimension vector as specified in Theorem 4.1, defined over an algebraically closed field $k$ of vanishing or sufficiently high characteristic. For every positive integer $m$, the Hilbert scheme of $m$ points $\mathcal{M}^{[m]}$ is again a moduli space of parabolic Higgs bundles, and the Hitchin morphism factors through the Hilbert-Chow map $\mathcal{M}^{[m]} \rightarrow \mathcal{M}^{(m)}$ to the symmetric product.

The theorem will be proved below as Theorem 5.1. In this article, we use the terminology parabolic Higgs bundle in the traditional sense (e.g., [14]), denoting a pair consisting of a parabolic vector bundle and a Higgs field, which is allowed to acquire order 1 poles at the marked points, such that the respective residues are nilpotent with respect to the flags coming from the parabolic structure. Boalch conjectured in [13, Remark 11.3] that the Hilbert scheme of $m$ points of a 2D moduli space of meromorphic Higgs bundles is again a moduli space of meromorphic Higgs bundles. It has been shown by Gorsky-Nekrasov-Rubtsov that Hilbert schemes of cotangent spaces of elliptic curves are moduli spaces of parabolic Higgs bundles [23, Section 5.1]. Theorem 1.2 thus extends their result to some other examples of 2D moduli spaces of parabolic Higgs bundles. It remains open whether our methods can be extended to treat the general case of Boalch's conjecture. Our treatment relies on some peculiarities of the tame parabolic case, for example, the description in terms of Higgs bundles on an orbifold (Proposition 2.21), for which no analogy for general meromorphic Higgs bundles is known to the author.

The work of Boalch and Biquard [11] revealed that moduli spaces of meromorphic Higgs bundles are naturally equipped with hyperkähler metrics (see also Nakajima's [39] in this context). In particular, the above theorem establishes the existence of a hyperkähler structure on the Hilbert schemes of certain noncompact hyperkähler surfaces.

The proofs of the Theorems 1.2 and 1.1 rely heavily on the machinery of the Fourier-Mukai transform (see Section 3.2). The use of these techniques in the theory of 
moduli is by no means original; the importance of the Fourier-Mukai transform for this field of geometry has already been realized in Mukai's paper [38]. While the arguments of Gorsky-Nekrasov-Rubtsov in [23, Section 5.1] were mainly based on a gauge theoretical foundation, a closer look at their work reveals that it uses a (relative) Fourier-Mukai transform. Below we give a more detailed description of our strategy to prove the result of [23]. We refer the reader to Theorem 5.1 for a more precise statement of our result.

Elliptic curves are self-dual abelian varieties. This means that an elliptic curve $E$ is canonically isomorphic to the moduli space $E^{\vee}$ of degree 0 line bundles on itself. In particular, we have a universal line bundle $\mathcal{P}$ on $E \times E$, called the Poincaré bundle. It was shown by Mukai [38] that $\mathcal{P}$ induces an equivalence of derived categories

$$
\Phi_{\mathcal{P}}: D_{\mathrm{coh}}^{b}(E) \cong D_{\mathrm{coh}}^{b}(E)
$$

of Fourier-Mukai type. While it is hard to picture the transform $\Phi_{\mathcal{P}}(\mathcal{F})$ of a general sheaf, we remark that the skyscraper sheaf $\mathcal{O}_{X}$ at $x \in E$ is sent to the corresponding line bundle $\mathcal{P}_{X}:=\left.\mathcal{P}\right|_{\{x\} \times E}$ on $E$.

By means of this self-duality and the Beauville-Narasimhan-Ramanan (BNR) see [7] correspondence (Proposition 2.2), the cotangent bundle $T^{*} E$ can be identified with the moduli space of degree 0 rank 1 Higgs bundles on $E$. Since $E$ is parallelizable, we obtain $T^{*} E \cong E \times \mathcal{A}$; here $\mathcal{A}$ is the affine space associated to the $1 \mathrm{D}$ vector space $H^{0}\left(E, \Omega_{E}^{1}\right)$. Base change now gives rise to the following autoduality property of the rank 1 Hitchin system

$$
\Phi_{\mathcal{P}}: D_{\mathrm{coh}}^{b}\left(T^{*} E\right) \cong D_{\mathrm{coh}}^{b}\left(T^{*} E\right)
$$

A point in $T^{*} E$, corresponding to the Higgs bundle $(E, \theta)$ is sent to the line bundle on the spectral curve, associated via the BNR correspondence. In particular, we see that autoduality for the rank 1 Hitchin system of an elliptic curve contains the identification of moduli spaces given by the BNR correspondence. We may see this as motivation to extract more information about moduli spaces of Higgs bundles from it. The structure sheaf of a length $n$ subscheme $T$ of $T^{*} E$ is a torsion sheaf $\mathcal{O}_{T}$. Its Fourier-Mukai transform $\Phi_{\mathcal{P}}\left(\mathcal{O}_{T}\right)$ turns out to be a coherent sheaf on $T^{*} E$, giving rise to a rank $n$ Higgs bundle on $E$ (Lemma 4.6). So far we have ignored the subscheme structure of $\mathcal{O}_{T}$, which is given by a surjection $\mathcal{O}_{T^{*} E} \rightarrow \mathcal{O}_{T}$. Applying the functor $\Phi_{\mathcal{P}}$ to this map, one can use a Serre duality argument to endow the Higgs bundle $\Phi\left(\mathcal{O}_{T}\right)$ with a parabolic structure (see the proof of Theorem 4.1). While the Higgs bundle $\Phi\left(\mathcal{O}_{T}\right)$ turns out to be semistable in general, the parabolic structure serves the purpose of stabilizing the Higgs bundle. 
Before explaining why this procedure gives rise to an equivalence of moduli spaces, we will illustrate the geometry of this map by analyzing it birationally. A generic length $n$ subscheme $T$ of $T^{*} E$ is given by $n$ distinct points $x_{1}, \ldots, x_{n}$. Each of these points corresponds to a rank 1 degree 0 Higgs bundle $\left(L_{1}, \theta_{1}\right), \ldots,\left(L_{n}, \theta_{n}\right)$ on $E$. The Higgs bundle $\Phi_{\mathcal{P}}(T)$ is given by the direct sum $(E, \theta):=\bigoplus_{i=1}^{n}\left(L_{i}, \theta_{i}\right)$. The parabolic structure is constructed by choosing a generic line within the zero fiber $E_{0}$. In this context, generic is meant to avoid the subspaces given by (direct sums of the) line bundles $L_{i}$. The isomorphism class in parabolic Higgs bundles turns out to be independent of this choice. The stabilizing effect of the parabolic structure alluded to above is captured by the fact that $(E, \theta)$ has no proper Higgs subbundle, which contains the chosen line.

We have constructed a natural map $f$ from the Hilbert scheme $\left(T^{*} E\right)^{[n]}$ to the moduli space $\mathcal{M}$ of rank $n$ parabolic Higgs bundles on $E$. It seems tempting to conclude the proof simply by claiming that the inverse of the equivalence $\Phi_{\mathcal{P}}$ yields an inverse to the map $f$. Unfortunately, it is not immediately obvious that $\Phi_{\mathcal{P}}^{-1}((E, \theta))$ of an arbitrary Higgs bundle gives rise to a complex supported in a single degree. On the other hand, we know that this will be the case for the parabolic Higgs bundles in the image of the map $f$. A short argument using properness of the Hitchin map can be applied to establish surjectivity of the map $f$, and therefore proves that $f$ is indeed an isomorphism of moduli spaces (see the end of the proof of Theorem 4.1).

The discussion above refers to the $\tilde{A}_{0}$-case, where we use the aforementioned description of parabolic moduli problems in terms of labeled graphs. In order to give the reader a feeling for the general argument, we repeat some of the explanation above for the case of $\tilde{D}_{4}$.

The geometric setup of the $\tilde{D}_{4}$-case is an elliptic curve $E$, together with the natural $\mathbb{Z} / 2 \mathbb{Z}$-action induced by the inverse map. The quotient of $E$ with respect to this action, is equivalent to a rational curve. The four fixed points give rise to four marked points on the copy of $\mathbb{P}^{1}$. A Higgs bundle on $E$, which is equivariant with respect to this action, corresponds via the dictionary described in Section 2.5, to a parabolic Higgs bundle on $\mathbb{P}^{1}$.

The derived equivalence

$$
\Phi_{\mathcal{P}}: D_{\mathrm{coh}}^{b}\left(T^{*} E\right) \cong D_{\mathrm{coh}}^{b}\left(T^{*} E\right),
$$

used above, allows us to associate to an element of the equivariant Hilbert scheme of $T^{*} E$ (see Section 3.3), an equivariant Higgs bundle on $E$. In the proof of Theorem 4.1, we check that this defines indeed an equivalence between the $\mathbb{Z} / 2 \mathbb{Z}$-equivariant Hilbert 
scheme of $T^{*} E$ and a 2D moduli space $\mathcal{M}$ of parabolic Higgs bundles. As above, this hinges on the properness of the Hitchin map.

The surface $\mathcal{M}$ is a moduli space for two different moduli problems: certain length $2 \mathbb{Z} / 2 \mathbb{Z}$-equivariant subschemes of $T^{*} E$ and certain rank 2 parabolic Higgs bundles on $\mathbb{P}^{1}$. Moreover, we show in Theorem 5.1 that the Hilbert scheme $\mathcal{M}^{[m]}$ is naturally equivalent to a certain moduli space of rank $2 m$ parabolic Higgs bundles on $\mathbb{P}^{1}$. The proof of this follows the same strategy as the proof of the $\tilde{A}_{0}$-case, which we described earlier. According to the McKay correspondence of Bridgeland-King-Reid [18], there is a canonical derived equivalence

$$
D_{\text {coh }}^{b}(\mathcal{M}) \cong D_{\text {coh }}^{b}\left(\left[T^{*} E /(\mathbb{Z} / 2 \mathbb{Z})\right]\right),
$$

which relies on $\mathcal{M}$ being a crepant resolution of the singular quotient of $T^{*} E$ with respect to the $\mathbb{Z} / 2 \mathbb{Z}$-action. We compose this derived equivalence with the equivariant version of Mukai's

$$
D_{\text {coh }}^{b}\left(\left[T^{*} E /(\mathbb{Z} / 2 \mathbb{Z})\right]\right) \cong D_{\text {coh }}^{b}\left(\left[T^{*} E /(\mathbb{Z} / 2 \mathbb{Z})\right]\right) .
$$

Thereby we obtain a way of relating the skyscraper sheaf of a closed point of $\mathcal{M}$ with an equivariant Higgs bundle on $E$. Given a length $m$ subscheme of the surface $\mathcal{M}$, we can take the transform of the underlying torsion sheaf with respect to this derived equivalence. We obtain an equivariant Higgs bundle on $E$, which corresponds via the dictionary of Section 2.5 to a parabolic Higgs bundle on $\mathbb{P}^{1}$. By virtue of Serre duality, the subscheme structure of the torsion sheaf gives rise to additional parabolic structure, which is described by means of the extended graph below. We will explain this in detail in Section 5.

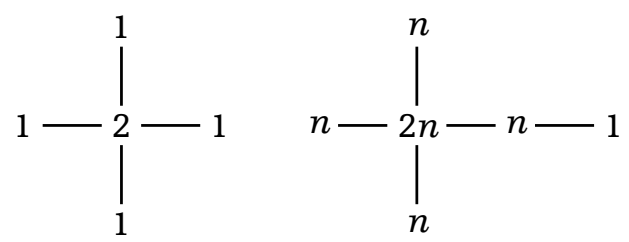

As has been mentioned above, the generic fiber of the Hitchin fibration $\chi: \mathcal{M} \rightarrow \mathcal{A}$ is a self-dual abelian variety. In particular, there exists a Zariski open subset $\mathcal{A}^{\text {sm }} \subset \mathcal{A}$ corresponding to smooth spectral curves and a line bundle $\mathcal{P}$ on $\mathcal{M}^{\mathrm{sm}} \times{ }_{\mathcal{A}^{\mathrm{sm}}} \mathcal{M}^{\mathrm{sm}}$ inducing a nontrivial autoequivalence

$$
D_{\text {coh }}^{b}\left(\mathcal{M}^{\mathrm{sm}}\right) \rightarrow D_{\text {coh }}^{b}\left(\mathcal{M}^{\mathrm{sm}}\right) .
$$

It is an important open problem to determine whether this equivalence extends to the whole base $\mathcal{A}$, where $\mathcal{M}$ is either the moduli space of semistable Higgs bundles or the 
full moduli stack. We refer the reader to [20, Conjecture 2.5] for a more precise statement of this conjecture. Recently, this has been achieved by Arinkin [3] over the locus of integral spectral curves.

To demonstrate the flexibility of our Theorems 1.1 and 1.2, we prove this socalled autoduality conjecture for the moduli space of Higgs bundles in all the cases given by Theorem 1.2 (see Theorem 5.5 for a proof).

Theorem 1.3. Let $\mathcal{M}$ be one of the 2D moduli spaces of parabolic Higgs bundles considered in Theorem 1.2. We denote its Hitchin base by $\mathcal{A}_{1}$. According to Theorem 1.2, the Hilbert scheme $\mathcal{M}^{[m]}$ is again a moduli space of parabolic Higgs bundles, with Hitchin base denoted by $\mathcal{A}_{m}$. There exists a derived equivalence of Fourier-Mukai type

$$
D_{\mathrm{coh}}^{b}\left(\mathcal{M}^{[m]}\right) \cong D_{\mathrm{coh}}^{b}\left(\mathcal{M}^{[m]}\right)
$$

defined relative to the Hitchin base $\mathcal{A}_{m}$ and extending the Fourier-Mukai transform over the locus of smooth spectral curves.

The main ingredient of the proof of Theorem 1.3 is the derived McKay correspondence as shown in [18]. Interestingly, both Arinkin's proof of the autoduality over the integral locus in [3] and our proof of Theorem 1.3 rely on Haiman's work on the $n$ !-conjecture [25].

All our results described above have a counterpart for moduli spaces of flat connections. The place of Mukai's equivalence is then taken by the derived Geometric Langlands Conjecture for $\mathrm{GL}_{1}$ (Theorem 4.7), which has been proved by Laumon [34] and Rothstein [48]. In the first case, there is no Hitchin map, and therefore no properness available. It is an old theorem of Weil (see Atiyah's exposition in [6]), which comes to the rescue. The existence of a flat connection in zero characteristic forces the degree of a vector bundle (and all of its indecomposable summands), which turns out to be what is needed for the inverse derived equivalence to give rise to an inverse map between the two moduli spaces. In positive characteristic, Weil's result ceases to hold, a loss which is compensated for by the return of a proper Hitchin map. For the sake of avoiding repetition, we refer the reader to Theorems 4.12, 5.2, and 5.6 instead.

\section{Higgs Bundles and Local Systems}

In this section, we will review the theory of Higgs bundles, local systems, and parabolic structures. From now on, $k$ denotes an algebraically closed field of either vanishing or 
sufficiently high characteristic. We assume that all schemes and related constructions are defined over $k$.

\subsection{Higgs bundles and the BNR correspondence}

In the following, we fix a smooth projective curve $X$ and a positive integer $n \in \mathbb{N}$. For a scheme $S$, we define the notion of an $S$-family of Higgs bundles below. Of particular importance is the case of a $k$-family, which simply is a Higgs bundle on $X$. We denote by $\Omega_{X}^{1}$ the cotangent sheaf on $X$ and by $p_{X}: X \times S \rightarrow X$ the canonical projection.

Definition 2.1. An $S$-family of Higgs bundles on $X$ consists of a locally free sheaf $\mathcal{E}$ of rank $n$ on $X \times S$ and a Higgs field given by a morphism of $\mathcal{O}_{X}$-modules

$$
\theta: \mathcal{E} \rightarrow \mathcal{E} \otimes p_{X}^{*} \Omega_{X}^{1}
$$

This gives rise to a moduli stack of Higgs bundles denoted by $\mathcal{M}_{\mathrm{Dol}}(X)$, which sends $S$ to the groupoid of $S$-families of Higgs bundles on $X$.

Higgs bundles on $X$ can be perceived as certain coherent sheaves on the cotangent bundle $T^{*} X$. This is known as the Beauville-Narasimhan-Ramanan correspondence [7] and was proved in full generality in [53, Lemma 6.8]. We will only need a weak version of the BNR correspondence, which is stated and proved below. The map $\pi: T^{*} X \rightarrow X$ is the canonical projection, and $\pi_{S}$ the base change

$$
T^{*}(X \times S / S) \rightarrow X \times S
$$

Proposition 2.2 (Weak BNR correspondence). There is a natural equivalence between the groupoid of $S$-families of Higgs bundles $(\mathcal{E}, \theta)$ and the groupoid of quasi-coherent $S$-flat sheaves $\mathcal{F}$ on the relative cotangent bundle $T^{*}(X \times S / S)$ satisfying

$$
\pi_{S, *} \mathcal{F}=\mathcal{E}
$$

Proof. Let us denote by $\Theta_{X}$ the tangent sheaf of $X$. The Higgs field $\theta$ of a Higgs pair $(\mathcal{E}, \theta)$ gives rise to a morphism of sheaves

$$
\mathcal{E} \otimes p_{X}^{*} \Theta_{X} \rightarrow \mathcal{E}
$$

which in turn induces a morphism

$$
\operatorname{Sym}^{\bullet} p_{X}^{*} \Theta_{X} \otimes \mathcal{E} \rightarrow \mathcal{E}
$$


This endows $\mathcal{E}$ with the structure of a module over the algebra

$$
\mathrm{Sym}^{\bullet} p_{X}^{*} \Theta_{X}=\pi_{S, *} \mathcal{O}_{T_{X \times S / S}^{*}} .
$$

Since $\pi_{S}$ is an affine morphism, this gives rise to an $S$-flat quasi-coherent sheaf $\mathcal{F}$ on $T_{X \times S / S}^{*}$, such that $\pi_{S, *} \mathcal{F}=\mathcal{E}$.

Vice versa, given an $S$-flat quasi-coherent sheaf $\mathcal{F}$ on $T_{X \times S / S}^{*}$ pushing-forward to a locally free sheaf $\pi_{S, *} \mathcal{F}=: \mathcal{E}$, we can define a Higgs field $\theta$ as follows: the push-forward $\mathcal{E}$ is endowed with the structure of an $\operatorname{Sym}^{\bullet} p_{X}^{*} \Theta_{X}$-module, in particular we have a map

$$
p_{X}^{*} \Theta_{X} \otimes \mathcal{E} \rightarrow \mathcal{E},
$$

giving rise to a Higgs field $\theta: \mathcal{E} \rightarrow \mathcal{E} \otimes p_{X}^{*} \Omega_{X}^{1}$.

If we perceive the Higgs field $\theta$ as a twisted endomorphism of $\mathcal{E}$, we see that the expression

$$
a(\lambda):=\operatorname{det}(\lambda-\theta)
$$

is well defined and is a polynomial

$$
\lambda^{n}+a_{n-1} \lambda^{n-1}+\cdots+a_{0},
$$

where $a_{i} \in H^{0}\left(X, \Omega_{X}^{\otimes(n-i)}\right)$.

Definition 2.3. Let $\mathcal{A}$ be the affine space associated to the vector space

$$
\bigoplus_{i=0}^{n-1} H^{0}\left(X, \Omega_{X}^{\otimes(n-i)}\right),
$$

it is called the Hitchin base. The morphism of stacks

$$
\chi_{\mathrm{Dol}}: \mathcal{M}_{\mathrm{Dol}}(X) \rightarrow \mathcal{A},
$$

sending a Higgs bundle $(\mathcal{E}, \theta)$ to the characteristic polynomial $a(\lambda)$ of $\theta$ is called the Hitchin morphism.

\subsection{Parabolic vector bundles}

As before, $X$ denotes a smooth projective curve of genus $g$. We assume that $D=n_{1} p_{1}+$ $\cdots+n_{k} p_{k}$ is an effective divisor on $X$, that is, $n_{i}>0$ for all $i$. The tuple $\hat{X}=(X, D)$ will 
be referred to as a weighted curve. We fix a positive integer $n \in \mathbb{N}$ and for $i=1, \ldots, k$ partitions

$$
n=\lambda_{i 0} \geq \cdots \geq \lambda_{i n_{i}}=0
$$

We will also write $l\left(\lambda_{i}\right)=n_{i}$ to denote the length of a partition. Following [26, Section 2.2], this numerical data will be encoded in the following diagram:

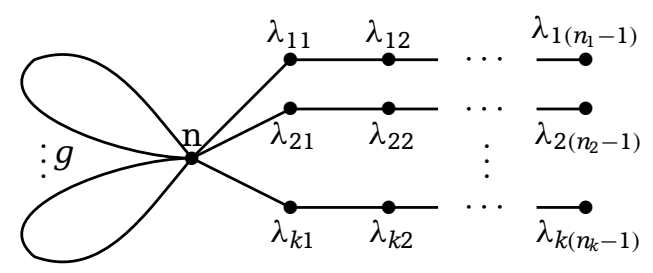

Let $S$ be a scheme. Below we define the notion of an $S$-family of parabolic vector bundles. We denote by $\iota_{i}: S \rightarrow X \times S$ the base change of the closed immersion

$$
p_{i}: \text { Spec } k \rightarrow X
$$

corresponding to the marked points $p_{i} \in X(k)$.

Definition 2.4. An $S$-family of quasi-parabolic vector bundles on $\hat{X}$ consists of a locally free sheaf $\mathcal{E}$ of rank $n$ on $X \times S$ together with flags

$$
0=F_{i n_{i}} \subset F_{i\left(n_{i}-1\right)} \subset \cdots \subset F_{i 0}=\iota_{i}^{*} \mathcal{E}
$$

of locally free subsheaves of $\iota_{i}^{*} \mathcal{E}$ of rank

$$
\operatorname{rk} F_{i j}=\lambda_{i j}
$$

such that the successive quotients are locally free. The moduli stack of quasi-parabolic vector bundles will be denoted by $\operatorname{Bun}(\hat{X})=\operatorname{Bun}\left(\hat{X}, n, \lambda_{\bullet}\right)$.

Given a vector bundle $E$ on $X$, a point $x \in X(k)$, and a subspace $L \subset E_{X}$ of the fiber $E_{X}=E / E(-X)$, we can define a locally free sheaf $E_{L}$ by the formula

$$
E_{L}:=\operatorname{ker}\left(E \rightarrow E_{X} / L\right),
$$


where $E \rightarrow E_{X} / L$ is the obvious map factoring through $E \rightarrow E_{X}$. This process can be reversed, since

$$
L=\operatorname{ker}\left(E_{X} \rightarrow \operatorname{coker}\left(E_{L} \rightarrow E\right)\right) .
$$

The process described above gives rise to an alternative description of quasiparabolic vector bundles as flags of locally free sheaves: For $i=1, \ldots, k$ and $j=-n+$ $1, \ldots, 0$, we define $E_{i j}$ to be the locally free subsheaf of $E$ given by $F_{j+n} \subset E_{p_{i}}$. For arbitrary $j \in \mathbb{Z}$, we can write $j=j^{\prime}+m$, where $-n<j^{\prime} \leq 0$ and $m \in \mathbb{Z}$, and define $E_{i j}:=$ $E_{i j^{\prime}} \otimes \mathcal{O}_{X}\left(m p_{i}\right)$. We conclude that there is an alternative description of parabolic bundles in terms of nested sequences of locally free sheaves [51, Section 3].

Lemma 2.5. The stack $\operatorname{Bun}\left(\hat{X}, n, \lambda_{\bullet}\right)$ is equivalent to the stack of families of $\mathbb{Z}^{k}$-indexed sequences of locally free sheaves $\left(V_{i}\right)_{i \in \mathbb{Z}^{k}}$ on $X$ satisfying

$$
V_{i} \subset V_{i+e_{j}}
$$

where $\left(e_{j}\right)$ denotes the canonical basis of $\mathbb{Z}^{k}$, and

$$
V_{i+n_{j} e_{j}}=V_{i} \otimes \mathcal{O}_{X}\left(p_{j}\right)
$$

The interpretation of parabolic vector bundles as sequences of locally free sheaves $\mathcal{V}=\left(V_{i}\right)_{i \in \mathbb{Z}^{k}}$ suggests a definition for the $d u a l$ quasi-parabolic vector bundle $\mathcal{V}^{\vee}$ given by the sequence

$$
V_{i}^{\vee}:=\left(V_{-i}\right)^{\vee}
$$

This description of the dual quasi-parabolic bundle is easily seen to be compatible with the following definition.

Definition 2.6. Let $\left(\hat{E}, F_{\text {.. }}\right)$ be a quasi-parabolic bundle on $\hat{X}$. The dual quasi-parabolic bundle $\hat{E}^{\vee}$ has underlying vector bundle $E^{\vee}$ and flag data given by

$$
F_{i j}^{\vee}=\operatorname{ker}\left(E_{X_{i}}^{\vee} \rightarrow F_{i(n-j)}^{\vee}\right) .
$$

Lemma 2.7. If $\hat{E}=\left(\hat{E}, F_{\bullet \bullet}\right)$ is a parabolic bundle on $\hat{X}$ corresponding to the sequence $\mathcal{V}=\left(V_{i}\right)_{i \in \mathbb{Z}^{k}}$, then the dual $\hat{E}^{\vee}$ corresponds to the dual sequence $\mathcal{V}^{\vee}=\left(\left(V_{-i}\right)^{\vee}\right)_{i \in \mathbb{Z}^{k}}$. 
Proof. For every $i=1, \ldots, k$, we denote by $e_{i}$ the canonical basis element in $\mathbb{Z}^{k}$. We need to compute

$$
\operatorname{ker}\left(E_{X_{i}}^{\vee} \rightarrow \operatorname{coker}\left(V_{-j e_{i}}^{\vee} \rightarrow E^{\vee}\right)\right)
$$

for $j=0, \ldots, n_{i}-1$. But $\operatorname{coker}\left(V_{-j e_{i}}^{\vee} \rightarrow E^{\vee}\right)=\operatorname{coker}\left(E \rightarrow V_{j e_{i}}\right)^{\vee}=F_{i(n-j)}^{\vee}$.

Stability conditions for quasi-parabolic vector bundles are parameterized by finite increasing sequences of positive real numbers $\left(\alpha_{i j}\right) \in[0,1)$, where $i=1, \ldots, k$ and $j=0, \ldots n_{i}-1$.

Definition 2.8. A pair consisting of a quasi-parabolic vector bundle and an increasing sequences of positive real numbers $\left(\alpha_{i j}\right) \in[0,1)$, where $i=1, \ldots, k$ and $j=0, \ldots n_{i}-1$, is called a parabolic vector bundle.

Following [14], we denote by $m_{i j}=\lambda_{i j}-\lambda_{i j+1}$ and define the parabolic degree of a parabolic vector bundle $\hat{E}$ to be

$$
\operatorname{deg} \hat{E}=\operatorname{deg} E+\sum_{i=1}^{k} \sum_{j=0}^{n_{i}-1} \alpha_{i j} m_{i j}
$$

The parabolic slope of $\hat{E}$ is given by

$$
\mu(\hat{E})=\frac{\operatorname{deg} \hat{E}}{\operatorname{rk} E} .
$$

Definition 2.9. A parabolic vector bundle $\hat{E}$ is said to be stable if for every proper subbundle $F$ of $E$ with the induced parabolic structure, we have

$$
\mu(\hat{F})<\mu(\hat{E})
$$

If we only have $\mu(\hat{F}) \leq \mu(\hat{E})$ for every proper subbundle $F$, we say that $\hat{E}$ is semistable.

\subsection{Parabolic Higgs bundles}

Definition 2.10. An $S$-family of parabolic Higgs bundles on $\hat{X}$ consists of an $S$-family of parabolic vector bundles $\left(\mathcal{E}, \mathcal{F}_{\text {.. }}\right)$ and a parabolic Higgs field given by a morphism of $\mathcal{O}_{X}$-modules

$$
\theta: \mathcal{E} \rightarrow \mathcal{E} \otimes p_{X}^{*} \Omega_{X}^{1}\left(p_{1}+\cdots+p_{k}\right)
$$


The latter is required to satisfy the condition that

$$
\operatorname{res}_{p_{i}} \theta\left(\mathcal{F}_{i j}\right) \subset \mathcal{F}_{i j+1}
$$

A parabolic Higgs bundle is stable (respectively, semistable) if the condition of Definition 2.9 is satisfied for all proper subbundles $F$ preserved by the Higgs field $\theta$. The moduli stack of parabolic Higgs bundles will be denoted by $\mathcal{M}_{\text {Dol }}(\hat{X})=\mathcal{M}_{\text {Dol }}\left(\hat{X}, n, \lambda_{\bullet}\right)$. The moduli space of stable parabolic Higgs bundles $\mathcal{M}_{\mathrm{Dol}}^{\mathrm{s}}$ is constructed as the rigidification of the open substack of stable Higgs bundles of $\mathcal{M}_{\text {Dol }}(X)$.

As before, there exists a Hitchin base

$$
\mathcal{A}:=\bigoplus_{i=0}^{n-1} H^{0}\left(X, \Omega_{X}^{i+1}\left(i p_{1}+\cdots+i p_{k}\right)\right)
$$

and a Hitchin morphism

$$
\chi_{\mathrm{Dol}}: \mathcal{M}_{\mathrm{Dol}}(\hat{X}) \rightarrow \mathcal{A}
$$

which sends a parabolic Higgs bundle to the characteristic polynomial of its Higgs field $\theta$. Using a method developed by Langton, Yokogawa shows in [57, Corollary 5.13 and Corollary 1.6] that the Hitchin map induces a proper map on the moduli space of parabolic Higgs bundles. In the case of Higgs bundles without parabolic structures, this is a theorem of Nitsure [42, Theorem 6.1].

Theorem 2.11 (Nitsure and Yokogawa). Let $\mathcal{M}_{\text {Dol }}(\hat{X})$ be a moduli space of semistable parabolic Higgs bundles of a fixed type. Then the Hitchin map

$$
\chi: \mathcal{M}_{\mathrm{Dol}}(\hat{X}) \rightarrow \mathcal{A}
$$

is proper.

\subsection{Parabolic local systems}

Closely related to the theory of Higgs bundles is the theory of local systems (i.e., vector bundles with a flat connection). The works of Hitchin [28] and Simpson [51, 52] have exhibited a natural hyperkähler structure on the moduli space of stable Higgs bundles defined over a complex curve. One obtains the moduli space of irreducible local systems by hyperkähler rotation. In particular, there is a canonical diffeomorphism relating the moduli space of stable Higgs bundles and irreducible local systems. 
Using the notation from Section 2.3, we define the notion of an $S$-family of parabolic local systems.

Definition 2.12. For a weighted curve $\hat{X}$, let $\omega_{i j} \in k$ be a tuple of scalars, where $i$ corresponds to marked points in $D \subset X$ and $0 \leq j \leq n_{i}-1$. An $S$-family of parabolic local system with eigenvalues $\left(\omega_{i j}\right)$ on $\hat{X}$ consists of an $S$-family of parabolic vector bundles $\left(\mathcal{E}, \mathcal{F}_{\text {.. }}\right)$ and a parabolic flat connection given by a morphism of $k$-linear sheaves

$$
\nabla: \mathcal{E} \rightarrow \mathcal{E} \otimes p_{X}^{*} \Omega_{X}^{1}\left(p_{1}+\cdots+p_{k}\right)
$$

satisfying the Leibniz identity and the condition that

$$
\operatorname{res}_{p_{i}} \nabla\left(\mathcal{F}_{i j}\right) \subset \mathcal{F}_{i j}
$$

with eigenvalues of the residue at $x_{i}$ is given by the scalars $\omega_{i j}$ on the $j$ th graded of $\iota_{i}^{*} \mathcal{E}$. A parabolic local system is stable (respectively, semistable) if the condition of Definition 2.9 is satisfied for all proper subbundles $F$ preserved by the connection $\nabla$. The moduli stack of parabolic local systems will be denoted by $\mathcal{M}_{\mathrm{dR}}(\hat{X})=\mathcal{M}_{\mathrm{dR}}\left(\hat{X}, n, \lambda_{\bullet}\right)$. If $\hat{X}=X$, that is, there are no marked points, we will simply speak of local systems on the curve $X$. The moduli space of stable parabolic local systems $\mathcal{M}_{\mathrm{dR}}^{\mathrm{s}}$ is constructed as the rigidification of the open substack of stable local systems of $\mathcal{M}_{\mathrm{dR}}(X)$.

In most cases, we will pick the eigenvalues $\omega_{i j}$ to be given by the canonical weights $\alpha_{i j}$.

\subsection{Orbifolds and parabolic structures}

Given a weighted curve $\hat{X}$, we can associate to it an orbifold $\tilde{X}$, assuming that the characteristic of $k$ is zero or large enough. We emphasize that the word orbifold refers to a smooth Deligne-Mumford stack [35, Definition 4.1] in our context.

The orbifold $\tilde{X}$ is defined by the following glueing data: let $\mathbb{D}_{X}$ denote the formal disk Spec $\hat{\mathcal{O}}_{X}$ around a point $x \in X(k)$. Given an effective divisor $D \subset X$ represented by the effective linear combination $n_{1} p_{1}+\cdots+n_{k} p_{k}$, we let $\mathbb{D}_{i}:=\mathbb{D}_{p_{i}}$ and

$$
U:=X-D
$$

The fiber product $U \times_{X} \mathbb{D}_{i}$ is given by the punctured formal disk

$$
\mathbb{D}_{i}^{\bullet}:=\operatorname{Frac} \hat{\mathcal{O}}_{X}
$$


Let us denote by

$$
[n]: \operatorname{Spec} k((t)) \rightarrow \operatorname{Spec} k((t))
$$

the faithfully flat morphism given by $t \mapsto t^{n}$. Note that this is an étale morphism if and only if the characteristic $p$ of $k$ does not divide $n$. Picking a formal coordinate $t_{i}$ around every point $p_{i}$, we obtain morphisms

$$
\left[n_{i}\right]: \quad \mathbb{D}_{i}^{\bullet} \rightarrow \mathbb{D}_{i}^{\bullet}
$$

for every $i=1, \ldots, n$.

On the disk $\mathbb{D}_{i}$, we define the obvious action of the group $\mu_{n_{i}}$ of $n_{i}$ th roots of unity by multiplication. Using this action, we glue the quotient stacks $\left[\mathbb{D}_{i} / \mu_{n_{i}}\right]$ back to $U$ using the $\mu_{n_{i}}$-equivariant maps induced by the $\left[n_{i}\right]$

$$
\mathbb{D}_{i}^{\bullet} \stackrel{\left[n_{i}\right]}{\rightarrow} \mathbb{D}_{i}^{\bullet} \rightarrow U
$$

According to [5, Theorem 6.1], this defines an algebraic stack independently of the characteristic $p$ of $k$. Nonetheless, this is a smooth Deligne-Mumford stack if either $p=0$ or $\operatorname{gcd}\left(p, n_{i}\right)=1$ for all $i$.

Assumption 2.13. The field $k$ is algebraically closed and its characteristic $p$ is either zero or satisfies $\operatorname{gcd}\left(p, n_{i}\right)=1$ for all $i$.

It is a result of Furuta-Steer [22, Section 5] that vector bundles on the orbifold $\tilde{X}$ translate into parabolic vector bundles on the weighted curve $\hat{X}$, where the weights are chosen to be the canonical weights $\alpha_{i j}:=\frac{j}{n_{i}}$. Nasatyr-Steer [40, Section 5A] discuss the analogous result for Higgs bundles. The local systems case is treated in [12] by BiswasHeu. In the remaining part of this subsection, we explain how this is proved in the realm of algebraic geometry instead of the analytic theory of Riemann surfaces used in [22,40].

Orbicurves as considered here can also be seen as certain root stacks associated to weighted curves (and this is what we will be doing implicitly). The correspondence described above is, therefore, reminiscent of a correspondence between parabolic vector bundles and vector bundles on root stacks, as established by Borne [15]. We refer the reader to [15] for the algebraic proofs of the statements below.

The correspondence between vector bundles on the orbifold $\tilde{X}$ and parabolic bundles on $\hat{X}$ is based on the following two observations. The natural morphism

$$
\tau: \tilde{X} \rightarrow X
$$


realizes $X$ as the coarse moduli stack for the Deligne-Mumford stack $\tilde{X}$. The second observation is that the functor $\tau_{*}$ from quasi-coherent sheaves on $\tilde{X}$ to quasi-coherent sheaves on $X$ is not faithful. Nonetheless, it sends a vector bundle $\tilde{E}$ on $\tilde{X}$ to a vector bundle $E:=\tau_{*} \tilde{E}$, since every torsion-free sheaf on a smooth curve is locally free.

Example 2.14. Let $\mu_{r}$ be the cyclic group $r$ th roots of unity. It acts on $\mathbb{D}:=$ Spec $k \llbracket t \rrbracket$ via $\xi \cdot t=\xi t$, where $\xi$ is an $r$ th root of unity. If $\tilde{X}$ is the quotient stack

\section{$\left[\mathbb{D} / \mu_{r}\right]$,}

we can identify the coarse moduli space $X$ with $\operatorname{Spec} k \llbracket t^{r} \rrbracket$. The functor $\tau_{*}$ sends a $\mu_{r}$-equivariant $k \llbracket t \rrbracket$-module $M$ to the $k \llbracket t^{r} \rrbracket$-module $M^{\mu_{r}}$.

To reconcile the loss of information under the map $\tilde{E} \mapsto E$, we define a $\mathbb{Z}$-indexed sequence of line bundles $\left(L_{i}\right)_{i \in \mathbb{Z}}$ for every orbifold point of the orbifold $\tilde{X}$, satisfying

$$
L_{i} \subset L_{i+1},
$$

for all $i \in \mathbb{Z}$, and send $\tilde{E}$ to the parabolic vector bundle associated to the filtered locally free sheaf $\left(\tau_{*}\left(\tilde{E} \otimes L_{i}\right)\right)_{i \in \mathbb{Z}}$.

Definition 2.15. Let $\hat{X}$ be a weighted curve and $\tilde{X}$ be the associated orbicurve. For every marked point $p_{i}$ of $\hat{X}$, we pick an $n$th root $L_{i 1}$ of $\tau^{*} \mathcal{O}_{X}\left(p_{j}\right)$. The line bundle $L_{i j}$ is defined to be

$$
L_{i j}:=L_{i 1}^{j}
$$

The existence of $L_{i 1}$ can be seen locally on $X$ using the notation of Example 2.14. Let $x$ be the origin of the disk $\mathbb{D}$. Since $\tau^{*} \mathcal{O}_{X}(x)$ is given by the $k \llbracket t \rrbracket$-module $t^{-n} k \llbracket t \rrbracket$, we see that $t^{-1} k \llbracket t \rrbracket$ is an $n$th root of $\tau^{*} \mathcal{O}_{X}(x)$.

By a formal-disk argument, we can show the following remark.

Remark 2.16. Let $n_{i}$ denote the order of the stabilizer group of the point $p_{i}$, respectively, the weight of $p_{i}$. Then, we have $\tau^{*} \mathcal{O}_{X}\left(p_{i}\right)=L_{i n_{i}}$.

Using this remark and Lemma 2.5, it is a consequence of the projection formula

$$
\tau_{*} \tau^{*} \mathcal{O}_{X}\left(p_{i}\right) \cong \mathcal{O}_{X}\left(p_{i}\right) \otimes \tau_{*} \mathcal{O}_{\tilde{X}} \cong \mathcal{O}_{X}\left(p_{i}\right)
$$


that the sequence of locally free sheaves

$$
E_{i j}:=\tau_{*}\left(\tilde{E} \otimes L_{i j}\right)
$$

gives rise to a parabolic vector bundle $\hat{E}:=\left(E, F_{\bullet \bullet}\right)$ on $\hat{X}$. We denote the map sending an orbibundle $\tilde{E}$ to the parabolic bundle $\hat{E}$ by $A$.

Proposition 2.17 (Furuta-Steer). The association

$$
A: \tilde{E} \mapsto \hat{E}
$$

described above gives rise to an equivalence of groupoids of vector bundles on the orbicurve $\tilde{X}$ and parabolic vector bundles on the weighted curve $\hat{X}$.

As a parabolic bundle is obtained from an orbibundle by push-forward, one should expect the respective degrees of the bundles to be related. A Riemann-Roch computation (using [56, Corollary 4.14] for Deligne-Mumford stacks) reveals the precise relation between the two degrees.

Lemma 2.18. Under the equivalence of Proposition 2.17, the degree of an orbibundle $\tilde{E}$ is equal to the parabolic degree of the parabolic bundle $\hat{E}$ with respect to the called canonical weights $\alpha_{i j}:=\frac{j}{n_{i}}$.

In the following remark, we make the above correspondence more explicit using the notation of Example 2.14.

Remark 2.19. Let $E$ be a $\mu_{r}$-equivariant vector bundle on $\mathbb{D}$. The projection formula implies that we have

$$
E^{\Gamma} \otimes \mathcal{O} / \mathcal{O}(-X) \cong\left(E \otimes \mathcal{O} / L^{-r}\right)^{\Gamma}
$$

Using this, we may identify the corresponding parabolic vector bundle $\hat{E}$ on $\hat{\mathbb{D}}$ with the one given by the vector bundle $E^{\Gamma}$ together with the flags

$$
F_{i}:=\left(E \otimes L^{-i} / L^{-r}\right)^{\Gamma} \subset\left(E \otimes \mathcal{O} / L^{-r}\right)^{\Gamma} \cong E^{\Gamma} \otimes \mathcal{O} / \mathcal{O}(-X)
$$

The next lemma and its proof should clarify how the equivariant structure of an vector bundle on an orbicurve is translated into the flag data of a parabolic vector bundle. 
Lemma 2.20. Let $\Gamma=\mu_{r}$ be the finite cyclic group of order $r$ acting on $\mathbb{D}=\operatorname{Spec} k \llbracket t \rrbracket$ through $\xi \cdot t=\xi t$, where $\xi$ is an $r$ th root of unity. Then the isomorphism classes of rank $n$ parabolic vector bundles on the weighted curve $\widehat{[\mathbb{D} / \Gamma]}$ correspond to isomorphism classes of representations of $\Gamma$ on an $n$-dimensional vector space. The regular representation of $\Gamma$ corresponds to a rank $r$ vector bundle with parabolic structure given by a complete flag.

Proof. Let us denote by $\chi$ the character associated to the zero fiber of the line orbibundle $L$. By assumption, we have $\chi(\xi)=\xi^{-1}$. If $E$ is a bundle on $[\mathbb{D} / \Gamma]$ and $\left(E_{i}\right)_{i \in \mathbb{Z}}$ denotes the corresponding parabolic bundle on $\widehat{[\mathbb{D} / \Gamma]}$. A section $s$ of $E_{i}$ nonvanishing at $0 \in \mathbb{D}$ corresponds to a $\Gamma$-invariant section of $E \otimes L^{i}$. This gives rise to an eigenline in $E_{0}$ on which $\Gamma$ acts by $\chi^{-i}$.

Vice versa given an eigenline $\langle v\rangle \subset(E)_{0}$ on which $\Gamma$ acts by $\chi^{k}$, this gives rise to an eigenline in $\left(E \otimes L^{-k}\right)_{0}$, on which $\Gamma$ acts trivially. This in turn gives rise to a section of $E_{k}$. We see that the parabolic structure encodes the $\Gamma$-action on the zero fiber $E_{0}$.

To verify the last assertion, we only have to observe that the regular representation of $\Gamma$ is the direct sum

$$
\bigoplus_{k=0}^{r} V_{\chi^{k}},
$$

where $V$ is a $1 \mathrm{D}$ vector space with $\Gamma$ acting on it through the character specified in the subscript.

As a next step, we investigate what happens to extra structures like a Higgs field or a connection under the transition $\tilde{E} \mapsto \hat{E}$. Definitions 2.1 and 2.12 are étale local in nature with respect to the curve $X$, and in particular, this allows us to make sense of Higgs bundles and local systems on an orbicurve $\tilde{X}$.

Proposition 2.21 (Nasatyr-Steer, Biswas-Heu). Under the correspondence of Proposition 2.17, a Higgs field $\tilde{\theta}$ on an orbibundle $\tilde{E}$ gets transformed to a parabolic Higgs field $\hat{\theta}$ on $\hat{E}$. Similarly, a flat connection $\tilde{\nabla}$ is sent to a parabolic flat connection $\hat{\nabla}$ with eigenvalues $\omega_{i j}$ given by the canonical weights. This defines a natural equivalence of groupoids between $S$-families of Higgs bundles (respectively, local systems) on the orbicurve $\tilde{X}$ and $S$-families of parabolic Higgs bundles (respectively, parabolic local systems) on the weighted curve $\hat{X}$. 


\section{Derived Equivalences}

This section is a collection of technical results on derived categories that will be of use later. The geometrically minded reader is encouraged to skip it and come back to it as required.

\subsection{Derived categories}

We begin by reviewing the theory of quasi-coherent sheaves and their derived categories on stacks. A good summary of this theory, together with theoretical justification for some of the definitions given below, is contained in [4, Section 2].

The data of a quasi-coherent sheaf $F$ on a prestack $\mathcal{X}$ are equivalent to a collection of quasi-coherent sheaves $F_{U \rightarrow \mathcal{X}}$ for every affine scheme $U$ with a morphism $U \rightarrow \mathcal{X}$, in a way compatible with pullback. This compatibility condition stipulates the existence of isomorphisms

$$
\phi_{V \rightarrow U}: \psi^{*} F_{U \rightarrow \mathcal{X}} \rightarrow F_{V \rightarrow \mathcal{X}}
$$

for every morphism $\psi: V \rightarrow U$ of $\mathcal{X}$-schemes, which are required to obey a compatibility law of their own. In the language of category theory, we have exhibited the category of quasi-coherent sheaves on $\mathcal{X}$ as the 2-limit of the categories $\mathrm{QCoh}(U)$ of quasi-coherent sheaves on $U$

$$
\operatorname{OCoh}(\mathcal{X}):=\lim _{U \in \operatorname{Aff} / \mathcal{X}} \operatorname{QCoh}(U)
$$

If $\mathcal{X}$ is an algebraic stack, it is possible to replace the above 2-limit by a less intimidating one. Let $Y \rightarrow \mathcal{X}$ be an atlas, that is, a smooth surjective morphism, where $Y$ is a scheme. Faithfully flat descent theory implies that $\operatorname{QCoh}(\mathcal{X})$ is equivalent to the 2-limit

$$
\mathrm{QCoh}(\mathcal{X}) \cong \lim [\mathrm{QCoh}(Y) \rightrightarrows \mathrm{OCoh}(Y \times \mathcal{X} Y) \rightrightarrows \mathrm{OCoh}(Y \times \mathcal{X} Y \times \mathcal{X} Y)]
$$

This 2-limit amounts to the simple fact that the data of a quasi-coherent sheaf on $\mathcal{X}$ is equivalent to a quasi-coherent sheaf $F_{Y}$ on the atlas $Y$ endowed with descent data. In the special case that $\mathcal{X}$ is a global quotient stack $[Y / G]$, where $G$ is a smooth algebraic group scheme, this descent data amounts to a $G$-equivariant structure on $F_{Y}$ [21, Definition I.3.46].

Below we give a definition of the bounded derived category of coherent sheaves $D_{\text {coh }}^{b}(\mathcal{X})$ on a stack $\mathcal{X}$. In the cases of interest to us this definition is equivalent to the one given in [35], but in the case of the unbounded derived category $D_{\text {qcoh }}(\mathcal{X})$ of quasicoherent sheaves we prefer to use a definition requiring slightly more machinery. 
Definition 3.1. Let $\mathcal{X}$ be a quasi-compact algebraic stack with affine diagonal and atlas $Y \rightarrow X$. We define the bounded derived category $D_{\text {coh }}^{b}(\mathcal{X})$ of coherent sheaves on $\mathcal{X}$ to be the full subcategory of the derived category of $\operatorname{QCoh}(\mathcal{X})$ of complexes $F^{\bullet}$ whose cohomology sheaves are coherent when pulled back to $Y$ and vanish for almost all degrees.

It is a well-known fact that the nonfunctoriality of cones leads to technical complications in the theory of derived categories. For instance, it is not possible to obtain $D_{\text {qoh }}(\mathcal{X})$ as a 2-limit of the derived categories $D_{\text {qcoh }}(U)$ for affine schemes $U \rightarrow \mathcal{X}$ as we did it for the abelian category above. And neither is the category of $G$-equivariant objects in $D_{\mathrm{qcoh}}(Y)$ equivalent to the derived category of the quotient stack $[Y / G]$. This defect of $D_{\text {qcoh }}(\mathcal{X})$ can be fixed by replacing the derived category by an enhancement, that is, a closely related object, from which $D_{\mathrm{qcoh}}(\mathcal{X})$ can be fully recovered, but which possesses a functorial construction of cones. One way to do this is by using the theory of stable $\infty$-categories [36]. Every affine scheme $U$ has an associated stable $\infty$-category $Q C(U)$, whose homotopy category is the derived category of quasi-coherent sheaves on $U$. For a prestack $\mathcal{X}$, one defines $O C(\mathcal{X})$ as the homotopy limit of $\infty$-categories

$$
O C(\mathcal{X}):=\lim _{U \in \operatorname{Aff} / \mathcal{X}} O C(U),
$$

in analogy with the definition of the category of quasi-coherent sheaves $\mathrm{OCoh}(\mathcal{X})$ given at the beginning of this section.

Definition 3.2. Let $\mathcal{X}$ be an algebraic stack, the derived category of quasi-coherent sheaves $D_{\mathrm{qcoh}}(\mathcal{X})$ is defined to be the homotopy category of the stable $\infty$-category $Q C(\mathcal{X})$.

Whenever possible, we will formulate proofs in the language of derived categories, but complementing our presentation by using stable $\infty$-categories. The inherent functoriality in the language of stable $\infty$-categories allows straightforward constructions, which would be more intricate in the world of triangulated categories. We demonstrate this principle with an easy lemma, which lies at the heart of our treatment of the autoduality conjecture in the special cases considered here (Theorems 4.13 and 5.5). A second proof, avoiding stable $\infty$-categories, will be supplied as Lemma 3.8.

Lemma 3.3. Let $X$ and $Y$ be two schemes, endowed with an action of an abstract finite group $\Gamma$; we assume that there is an equivalence of $\infty$-categories

$$
Q C(X) \cong Q C(Y)
$$


which is $\Gamma$-equivariant. This induces an equivalence

$$
O C([X / \Gamma]) \cong O C([Y / \Gamma]) .
$$

Proof. Since $X \rightarrow[X / \Gamma]$ is an atlas for the stack $[X / \Gamma]$, it is possible to write $O C([X / \Gamma])$ as the homotopy limit

$$
\lim _{J \in \Delta^{\mathrm{op}}} O C\left(X^{[J]}\right)
$$

where $\Delta$ denotes the category of finite nonempty ordered sets and

$$
X^{[J]}:=X \times \Gamma^{J}
$$

Let $B \Gamma$ be the nerve of the groupoid associated to the group $\Gamma$. The $\Gamma$-action on $X$ induces an action on $Q C(X)$, which is encoded by an $\infty$-functor from $B \Gamma$ to the $\infty$-category of $\infty$-categories

$$
\text { act : } B \Gamma \rightarrow \infty-\text { Cat. }
$$

The above homotopy limit can be rewritten as

$$
\lim _{B \Gamma} \operatorname{act}
$$

which is a purely $\infty$-categorical construction, and therefore depends only on the $\infty$-category $O C(X)$ and the $\Gamma$-action up to equivalence. In general, we refer to such a limit as the $\infty$-category of $\Gamma$-equivariant objects in a $\infty$-category. As the equivalence $O C(X) \cong O C(Y)$ respects the $\Gamma$-action, we obtain that the $\infty$-categories of $\Gamma$-equivariant objects in $O C(X)$ and $O C(Y)$ must be equivalent. In particular, we have

$$
O C([X / \Gamma]) \cong Q C([Y / \Gamma]) .
$$

Even more generally, for an $\infty$-groupoid $G$, which is pointed and connected, and an $\infty$-functor act : $G \rightarrow \infty-$ Cat, we should think of the homotopy $\operatorname{limit} \mathcal{C}^{\Gamma}:=\lim _{G}$ act as an $\infty$-category of $G$-equivariant objects in an $\infty$-category $\mathcal{C}$. If $\mathcal{C}$ is stable (in particular, its homotopy category is triangulated), then so is $\mathcal{C}^{\Gamma}$ according to [36, Theorem 5.4]. In [54], an alternative linearization procedure is described for triangulated categories having a strongly pre-triangulated dg-model. Using this definition of linearization, Sosna also obtains an analog of Lemma 3.3 in [54].

\subsection{Fourier-Mukai transform}

Let $\mathcal{X}, \mathcal{Y}$, and $\mathcal{Z}$ be algebraic stacks that we assume to be quasi-compact and having affine diagonal. Let $\mathcal{X} \rightarrow \mathcal{Z}$ and $\mathcal{Y} \rightarrow \mathcal{Z}$ be morphisms of stacks and $K \in D_{\text {qcoh }}(\mathcal{X} \times \mathcal{Z} \mathcal{Y})$ be 
a complex on the fiber product $\mathcal{X} \times_{\mathcal{Z}} \mathcal{Y}$. If we denote by $p_{\mathcal{X}}: \mathcal{X} \times_{\mathcal{Z}} \mathcal{Y} \rightarrow \mathcal{X}$ the canonical projection, and similarly for $p_{y}$, we obtain an exact functor

$$
\Phi_{K}: D_{\mathrm{qcoh}}(\mathcal{X}) \rightarrow D_{\mathrm{qcoh}}(\mathcal{Y})
$$

which sends the complex of sheaves $F \in D_{\mathrm{qcoh}}(\mathcal{X})$ to

$$
\Phi_{K}(F):=R p_{\mathcal{Y}, *}\left(L p_{\mathcal{X}}^{*} F \otimes^{L} K\right)
$$

Functors between derived categories of this type are referred to as (generalized) FourierMukai transforms and were introduced by Mukai [38]. The following statement is proved as in [38], but using a slightly more general base change formula (e.g., [8, Proposition 3.10]). The proof can also be extracted from the proof of Lemma 3.7.

Lemma 3.4. Let $\mathcal{X}, \mathcal{Y}$, and $\mathcal{Z}$ be $\mathcal{W}$-stacks. We assume that all of these stacks are algebraic, quasi-compact, and have affine diagonal; moreover, we require that $\mathcal{X} \rightarrow \mathcal{W}$, $\mathcal{Y} \rightarrow \mathcal{W}$, and $\mathcal{Z} \rightarrow \mathcal{W}$ are representable flat morphisms. For $L \in D_{\mathrm{qcoh}}(\mathcal{X} \times \mathcal{W} \mathcal{Y})$ and $K \in$ $D_{\mathrm{qcoh}}(\mathcal{Y} \times \mathcal{W} \mathcal{Z})$, we define

$$
L * K:=R p_{\mathcal{X}, *}\left(L p_{\mathcal{X Y}}^{*} \otimes^{L} L p_{\mathcal{Y}}^{*} K\right)
$$

There exists a natural equivalence between the functors $\Phi_{K} \circ \Phi_{L}$ and $\Phi_{L * K}$.

As we are mainly dealing with generalized Fourier-Mukai functors, that is, integral kernels living on a fiber product $\mathcal{X} \times \mathcal{Z} \mathcal{Y}$, we have to investigate how the kernel changes if we replace the base $\mathcal{Z}$ along a morphism $\mathcal{Z} \rightarrow \mathcal{W}$. The behavior of integral kernels under this change of base stack is expressed in the well-known lemma below, which is proved by application of the projection formula (e.g., [8, Proposition 3.10]).

Lemma 3.5. Let $\mathcal{X}, \mathcal{Y}, \mathcal{Z}$, and $\mathcal{W}$ be algebraic stacks that are quasi-compact and have affine diagonal. We assume that $\mathcal{X}$ and $\mathcal{Y}$ are $\mathcal{Z}$-stacks and that there is a schematic morphism $\mathcal{Z} \rightarrow \mathcal{W}$. Let

$$
f:=\left(p_{\mathcal{X}}, p_{\mathcal{Y}}\right): \mathcal{X} \times_{\mathcal{Z}} \mathcal{Y} \rightarrow \mathcal{X} \times_{\mathcal{W}} \mathcal{Y}
$$

be the canonical morphism, and $K \in D_{\mathrm{qcoh}}\left(\mathcal{X} \times_{\mathcal{Z}} \mathcal{Y}\right)$. Then the Fourier-Mukai transform $\Phi_{K}$ is naturally equivalent to $\Phi_{R f_{*} K}$.

We will also have to understand the behavior of Fourier-Mukai transform with respect to base change. 
Lemma 3.6. Let $\mathcal{X}, \mathcal{Y}, \mathcal{Z}$, and $\mathcal{W}$ be perfect algebraic stacks that are quasi-compact and have affine diagonal. We assume that $\mathcal{X}$ and $\mathcal{Y}$ are flat $\mathcal{Z}$-stacks, and that there is a schematic morphism $\pi: \mathcal{W} \rightarrow \mathcal{Z}$. Every Fourier-Mukai equivalence

$$
\Phi_{K}: D_{\mathrm{qcoh}}(\mathcal{X}) \cong D_{\mathrm{qcoh}}(\mathcal{Y})
$$

relative to $\mathcal{Z}$ induces a Fourier-Mukai equivalence relative to $\mathcal{W}$

$$
\Phi_{\pi^{*} K}: D_{\mathrm{qcoh}}(\mathcal{X} \times \mathcal{Z} \mathcal{W}) \cong D_{\mathrm{qcoh}}(\mathcal{Y} \times \mathcal{Z} \mathcal{W})
$$

by pulling back the kernel $K$.

Proof. Let $L \in D_{\mathrm{qcoh}}(\mathcal{X} \times \mathcal{Z} \mathcal{Y})$ so that $K * L \cong \Delta_{*} \mathcal{O}_{\mathcal{X}}$ and $L * K \cong \Delta_{*} \mathcal{O}_{\mathcal{Y}}$. The base change formula implies that the same relations hold for $\mathcal{X} \times_{\mathcal{Z}} \mathcal{W}$ and $\mathcal{Y} \times \mathcal{Z} \mathcal{W}$.

The next lemma tells us that if $X_{i}$ and $Y_{i}$ are Fourier-Mukai partners for $i=1,2$, then products $X_{1} \times X_{2}$ and $Y_{1} \times Y_{2}$ are Fourier-Mukai partners. Using the formalism of stable $\infty$-categories, this is simply a consequence of [8, Theorem 1.2].

Lemma 3.7. For $i=1,2$, let $\mathcal{X}_{i}, \mathcal{Y}_{i}, \mathcal{Z}_{i}$, and $\mathcal{W}$ be perfect algebraic stacks that are quasicompact and have affine diagonal. We assume that $\mathcal{X}_{i}, \mathcal{Y}_{i}, \mathcal{Z}_{i}$ are $\mathcal{W}$-stacks and that the structural morphisms are flat. Let $D_{\mathrm{qcoh}}\left(\mathcal{X}_{i}\right) \cong D_{\mathrm{qcoh}}\left(\mathcal{Y}_{i}\right)$ be derived equivalences of Fourier-Mukai type, induced by integral kernels $K_{i} \in D_{\mathrm{qcoh}}\left(\mathcal{X}_{i} \times \mathcal{Z}_{i} \mathcal{Y}_{i}\right)$. Then $K_{1} \otimes^{L} K_{2}$ induces a derived equivalence

$$
D_{\mathrm{qcoh}}\left(\mathcal{X}_{1} \times \mathcal{W} \mathcal{X}_{2}\right) \cong D_{\mathrm{qcoh}}\left(\mathcal{Y}_{1} \times \mathcal{W} \mathcal{Y}_{2}\right)
$$

relative to $\mathcal{Z}_{1} \times_{\mathcal{W}} \mathcal{Z}_{2}$.

Proof. According to Lemma 3.6, we know that the equivalences $D_{\text {qcoh }}\left(\mathcal{X}_{i}\right) \cong D_{\text {qcoh }}\left(\mathcal{Y}_{i}\right)$ induce equivalences

$$
D_{\mathrm{qcoh}}\left(\mathcal{X}_{1} \times \mathcal{W} \mathcal{Y}_{1}\right) \cong D_{\mathrm{qcoh}}\left(\mathcal{X}_{2} \times \mathcal{W} \mathcal{Y}_{1}\right)
$$

and

$$
D_{\mathrm{qcoh}}\left(\mathcal{X}_{2} \times_{\mathcal{W}} \mathcal{Y}_{1}\right) \cong D_{\mathrm{qcoh}}\left(X_{2} \times_{\mathcal{W}} Y_{2}\right)
$$

By juxtaposition we obtain a derived equivalence

$$
D_{\mathrm{qcoh}}\left(\mathcal{X}_{1} \times \mathcal{W} \mathcal{Y}_{1}\right) \cong D_{\mathrm{qcoh}}\left(\mathcal{X}_{2} \times \mathcal{W} \mathcal{Y}_{2}\right)
$$


In order to obtain a better understanding of the integral kernel of this composition, we take a look at the following commutative diagram with Cartesian squares:

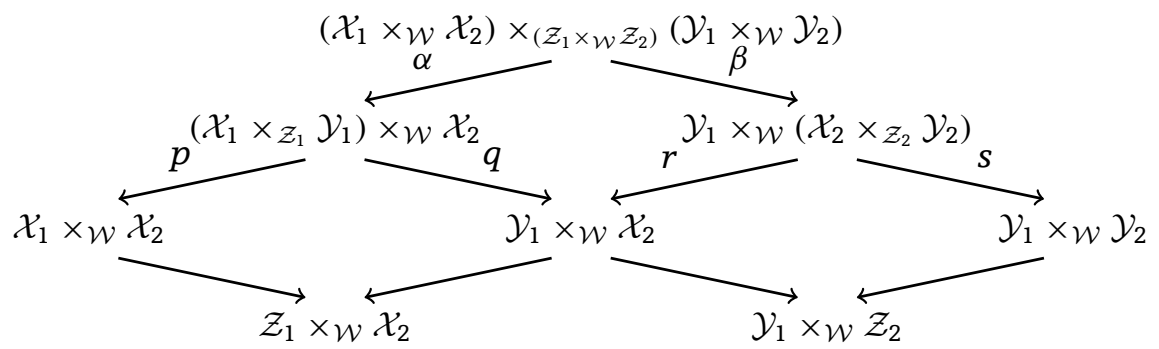

Let $M \in D_{\mathrm{qcoh}}\left(\mathcal{X}_{1} \times \mathcal{Y}_{1}\right)$, we denote by

$$
c:\left(\mathcal{X}_{1} \times \mathcal{Z}_{1} \mathcal{Y}_{1}\right) \times \mathcal{X}_{2} \rightarrow \mathcal{X}_{1} \times \mathcal{Z}_{1} \mathcal{Y}_{1}
$$

and

$$
d: \mathcal{Y}_{1} \times\left(\mathcal{X}_{2} \times \mathcal{Z}_{2} \mathcal{Y}_{2}\right) \rightarrow \mathcal{X}_{2} \times \mathcal{Z}_{2} \mathcal{Y}_{2}
$$

the canonical projection; the base change formula reveals now that

$$
R s_{*}\left(\left(L r^{*} R q_{*}\left(L p^{*} \otimes^{L} L c^{*} K_{1}\right)\right) \otimes^{L} L d^{*} K_{2}\right) \cong R s_{*}\left(R \beta_{*}\left(L \alpha^{*} L p^{*} M \otimes^{L} K_{1}\right) \otimes^{L} L d^{*} K_{2}\right) .
$$

Using the projection formula, we obtain

$$
R s_{*} R \beta_{*}\left(L \alpha^{*} L p^{*} M \otimes L \alpha^{*} L c^{*} K_{1} \otimes^{L} L \beta^{*} L d^{*} K_{2}\right)
$$

In particular, we see that the integral kernel is given by $K_{1} \otimes^{L} K_{2}$.

The lemma below is reminiscent from Lemma 3.3; it is formulated and proved in a more classical language, but using more restrictive assumptions.

Lemma 3.8. Let $X, Y$, and $Z$ be quasi-projective smooth $k$-varieties, proper, and flat over $Z$, endowed with the action of an abstract finite group $\Gamma$, such that the characteristic of $k$ does not divide $\Gamma$; we assume that there is a functor of Fourier-Mukai type

$$
\Phi_{K}: D_{\mathrm{coh}}^{b}(X) \rightarrow D_{\mathrm{coh}}^{b}(Y)
$$

given by an integral kernel $K \in D_{\mathrm{qcoh}}\left(X \times_{Z} Y\right)$ of finite Tor-dimension. Moreover, we assume that $K$ is endowed with a $\Gamma$-equivariant structure in the sense that $K \cong f^{*} L$ for

$$
L \in D_{\mathrm{coh}}^{b}\left([X / \Gamma] \times_{[Z / \Gamma]}[Y / \Gamma]\right),
$$


and the obvious map

$$
f: X \times_{Z} Y \rightarrow[X / \Gamma] \times_{[Z / \Gamma]}[Y / \Gamma]
$$

Then $\Phi_{K}$ is an equivalences of categories if and only if $\Phi_{L}$ is.

Proof. Let $W$ be a scheme with a $\Gamma$-action. We denote by

$$
f_{W}: W \rightarrow[W / \Gamma]
$$

the canonical morphism to the quotient stack. All these maps are faithfully flat, and therefore, we do not have to distinguish between $f_{W}^{*}$ and $L f_{W}^{*}$. For $L \in D_{\text {coh }}^{b}\left([X / \Gamma] \times_{[Z / \Gamma]}\right.$ $[Y / \Gamma]$ ), we denote the associated Fourier-Mukai transform by $\Phi_{[X / \Gamma][Y / \Gamma]}$ and similarly will $\Phi_{[X / \Gamma] Y}$ denote the functor $D_{\mathrm{coh}}^{b}([X / \Gamma]) \rightarrow D_{\mathrm{coh}}^{b}(Y)$ associated to the kernel obtained from $L$ via pullback along the obvious map, and so on.

The base change formula implies

$$
f_{Y}^{*} \Phi_{[X / \Gamma][Y / \Gamma]} \cong \Phi_{[X / \Gamma] Y} \cong \Phi_{X Y} f_{X}^{*}
$$

Since $f_{Y}$ and $f_{X}$ are faithfully flat, we conclude that $f_{Y}^{*}$ and $f_{X}^{*}$ are fully faithful functors. Together with $\Phi_{X Y}$ being fully faithful, we conclude that $\Phi_{[X / \Gamma][Y / \Gamma]}$ is fully faithful.

The functor $\Phi_{[X / \Gamma][Y / \Gamma]}$ has a right adjoint $\Psi_{[X / \Gamma][Y / \Gamma]}$ given by the integral kernel $L^{\vee} \otimes \omega$ on $[Y / \Gamma] \times_{[Z / \Gamma]}[X / \Gamma]$, where $\omega$ is a factor derived from a relative dualizing line bundle. This is seen by using the standard adjunctions together with Grothendieck-Serre duality. Replacing $\Phi$ by $\Psi$ above, we see that

$$
f_{X}^{*} \Psi_{[Y / \Gamma][X / \Gamma]}(F) \cong \Psi_{Y X}\left(f_{Y}^{*} F\right),
$$

for $F \in D_{\operatorname{coh}}^{b}([Y / \Gamma])$. Since $\Psi_{Y X}$ is a quasi-inverse to $\Phi_{X Y}$, we obtain from this relation that $\Psi_{[Y / \Gamma][X / \Gamma]}(F)=0$ if and only if $F=0$, again by virtue of the fact that $f_{X}$ and $f_{Y}$ are faithfully flat. Lemma 2.1 of [18] implies now that $\Phi_{[X / \Gamma][Y / \Gamma]}$ is an equivalence of categories.

We emphasize that every functor of Fourier-Mukai type $D_{\mathrm{qcoh}}(\mathcal{X}) \rightarrow D_{\mathrm{qcoh}}(\mathcal{Y})$ lifts to an $\infty$-functor $Q C(\mathcal{X}) \rightarrow Q C(\mathcal{Y})$. And for a large class of stacks, every functor between $\infty$-categories $Q C(\mathcal{X}) \rightarrow O C(\mathcal{Y})$ is obtained from a Fourier-Mukai transform [8, Theorem 1.2(2)]. For our purposes, it is therefore merely a matter of taste whether one utilizes the theory of stable $\infty$-categories or derived categories and functors of FourierMukai type. 


\subsection{The McKay correspondence}

In the paper [18], an important special case of the Fourier-Mukai transform has been considered to establish a form of the derived McKay correspondence. We denote by $X$ a smooth quasi-projective variety with an abstract finite group $\Gamma$ acting on it. Moreover, we assume that the characteristic of $k$ is zero or $p>|\Gamma|$. Let us denote by $Y \subset \operatorname{Hilb}^{|\Gamma|}[X / \Gamma]$ the scheme representing the functor given by $\Gamma$-equivariant subschemes

$$
Z \rightarrow X,
$$

such that there exists a surjection of $\Gamma$-equivariant sheaves $\mathcal{O}_{X} \rightarrow \mathcal{O}_{Z}$ and $\Gamma$ acts on $H^{0}\left(Z, \mathcal{O}_{Z}\right)$ as the regular representation. Moreover, we remove redundant irreducible components, so that we are left with the irreducible component containing the free $\Gamma$ orbits.

The fiber product $Y \times[X / \Gamma]$ is endowed with the structure sheaf $\mathcal{O}_{\mathcal{Z}}$ of the universal $\Gamma$-cluster $\mathcal{Z}$.

Theorem 3.9 ([18, Theorem 1.1]). We assume that the $\Gamma$-Hilbert scheme $Y$ of $X$ is smooth and satisfies the estimate

$$
\operatorname{dim} Y \times_{X / \Gamma} Y \leq \operatorname{dim} Y+1,
$$

where $X / \Gamma$ denotes the GIT quotient. Then the structure sheaf of the universal family $\mathcal{O}_{\mathcal{Z}}$ of $\Gamma$-clusters on

$$
Y \times[X / \Gamma]
$$

induces an equivalence of $k$-linear derived categories of Fourier-Mukai type

$$
D_{\mathrm{coh}}^{b}(Y) \cong D_{\mathrm{coh}}^{b}([X / \Gamma]) .
$$

In [18], a slightly more general theorem is proved for $k=\mathbb{C}$. The reason for this restriction is the use of the so-called New Intersection Theorem due to Roberts [47] and Peskine-Szpiro, which guarantees smoothness of $Y$. While this theorem holds in positive characteristic, [18] uses an addendum proved in [17] in the right generality. Nonetheless, in cases of interest to us, $Y$ will be already known to be smooth for different reasons.

In the next lemma, we observe how this Fourier-Mukai transform interacts with a given morphism $X \rightarrow S$. This will be important for our analysis of autoduality of the Hitchin fibration in Theorems 4.13 and 5.5. 
Lemma 3.10. Let $\pi: X \rightarrow S$ be a flat morphism of smooth quasi-projective varieties, endowed with the action of a finite group $\Gamma$, such that $\pi$ is $\Gamma$-equivariant. If $X$ satisfies the conditions of Theorem 3.9, $Y$ denotes the $\Gamma$-equivariant Hilbert scheme as before, and $S / \Gamma$ the GIT quotient, then the natural equivalence of derived categories

$$
D_{\mathrm{coh}}^{b}(Y) \cong D_{\mathrm{coh}}^{b}([X / \Gamma])
$$

is of Fourier-Mukai type relative to $S / \Gamma$.

Proof. We only need to check that $\mathcal{O}_{\mathcal{Z}}$ is supported on the fiber product

$$
Y \times_{S / \Gamma}[X / \Gamma]
$$

which one expects to be a consequence of the $\Gamma$-equivariance of $\pi$. To show this, we may cover $S / \Gamma$ by Zariski open affine subsets $U_{i}$ and cover $S$ by the fiber products

$$
S_{i}:=U_{i} \times_{S / \Gamma} S
$$

which are still affine, as $S \rightarrow S / \Gamma$ is finite. Using quasi-projectivity of $X$, we can cover $X \times{ }_{S} S_{i} \subset X$ by Zariski open affine subsets $V_{i}$, which are $\Gamma$-invariant. Henceforth, we may assume without loss of generality that $X=\operatorname{Spec} A$ and $S=\operatorname{Spec} D$ are affine varieties endowed with the action of an abstract group $\Gamma$.

Let now $C$ be another algebra endowed with the trivial $\Gamma$-action and $B$ be a $C$-flat quotient of $A \otimes C$ sitting in a short exact sequence

$$
0 \rightarrow I \rightarrow A \otimes C \rightarrow B \rightarrow 0
$$

such that $I$ is a $\Gamma$-invariant ideal of $A \otimes C$. In particular, this is a short exact sequence of $\Gamma$-modules. Moreover, we assume that $C \rightarrow A \otimes C \rightarrow B$ induces an isomorphism

$$
C \cong B^{\Gamma}
$$

This algebraic data encodes a $C$-point of the $\Gamma$-Hilbert scheme of $X$.

Since $A$ is a $D$-algebra, we obtain a natural morphism

$$
D^{\Gamma} \rightarrow B^{\Gamma}=C
$$

endowing $C$ with the structure of a $D^{\Gamma}$-algebra. Therefore, we see that on the $A \otimes C$-module $B$ the action of $D^{\Gamma}$ via $A$ agrees with the action via $C$. Thus, $B$ is actually a $A \otimes_{D^{\Gamma}} C$-module, which is what we wanted to show. 
An important example is given by Hilbert schemes of surfaces. To see how those relate to equivariant Hilbert schemes, we quote the following result of Haiman [25, Theorem 6], which is a corollary of his proof of the $n$ !-conjecture. (In [25], this theorem is stated for $k=\mathbb{C}$. The proof translates without problems to the more general case of characteristic zero or $p>n$. This is due to the fact that the main technical ingredient of Haiman's proof, the Polygraph Theorem, is already established in the required generality.)

Theorem 3.11 (Haiman). Let $X$ be a surface defined over a field of characteristic $p>n$ or zero. Let us denote by $X^{[n]}$ the Hilbert scheme of length $n$ subschemes and by $Y_{n}$ the $S_{n}$-Hilbert scheme of $X^{n}$ with respect to the natural group action of the symmetric group $S_{n}$ given by permuting factors. Then there is a natural isomorphism

$$
Y_{n} \cong X^{[n]} .
$$

Combining this result with Theorem 3.9, we obtain a well-known derived equivalence.

Corollary 3.12. If $X$ denotes a surface defined over a field of characteristic $p>n$ or zero, and $X^{[n]}$ denotes the Hilbert scheme of length $n$ subschemes, then we have a natural derived equivalence

$$
D_{\mathrm{coh}}^{b}\left(X^{[n]}\right) \cong D_{\mathrm{coh}}^{b}\left(\left[X^{n} / S_{n}\right]\right)
$$

Note that the required dimension estimate follows from the classical result of Briançon [16] and Iarrabino [29, Corollary 1] that for the punctual Hilbert scheme $\mathrm{Hilb}_{0}^{m} \mathbb{A}^{2}$ we have

$$
\operatorname{dim} \operatorname{Hilb}_{0}^{m} \mathbb{A}^{2}=m-1
$$

\section{Moduli Spaces of Dimension 2}

Let $Q$ be a Dynkin diagram, such that the corresponding affine Dynkin diagram $\tilde{Q}$ is comet-shaped (see Section 2.2). The only Dynkin diagrams satisfying this assumption are $A_{0}, D_{4}, E_{6}, E_{7}$, and $E_{8}$. In Section 2.3, we explained how comet-shaped graphs together with a dimension vector encode moduli problems for parabolic Higgs bundles. The graphs $\tilde{Q}$ listed above together with the basic imaginary root $\lambda$ are exactly the ones corresponding to the moduli spaces of parabolic Higgs bundles of dimension 2 which we will describe. 
The $A_{0}$-case is the simplest one, it describes Higgs bundles of rank 1 on an elliptic curve $E$, and the moduli space is $T^{*} E$. Nonetheless, there are many other examples of 2D moduli spaces of Higgs bundles that are somehow reminiscent of this one. To each graph describing a moduli space of parabolic Higgs bundles, we can associate a finite group $\Gamma$. For $D_{4}, E_{6}, E_{7}$, and $E_{8}$, these groups are $\mathbb{Z} / 2 \mathbb{Z}, \mathbb{Z} / 3 \mathbb{Z}, \mathbb{Z} / 4 \mathbb{Z}$, and $\mathbb{Z} / 6 \mathbb{Z}$. Let now $E$ be an appropriate elliptic curve with a $\Gamma$-action. In the $D_{4}$-case, $E$ is an arbitrary elliptic curve with $\mathbb{Z} / 2 \mathbb{Z}$ acting on it via $x \mapsto-x$. In all the other cases, the $\Gamma$-action stems from complex multiplication on the curve $E$, as has been explained in Section 1. This allows us to formulate the following folklore theorem, which will be proved in Section 4.2.

Theorem 4.1. We consider the weighted curve associated to the orbifold $[E / \Gamma]$, for the marked points away from $0 \in E$ we consider canonical weights. At the marked point corresponding to $0 \in E$, we work with the weights $\alpha_{i}:=\frac{i}{r}$ for $i<r-1$ and $1>\alpha_{r-1}>\frac{r-1}{r}$, where $r:=|\Gamma|$. The moduli space of stable parabolic Higgs bundles $\mathcal{M}_{\mathrm{Dol}}^{\mathrm{s}}(Q, \lambda)$ of orbifold degree 0 with respect to these weights is naturally isomorphic to the $\Gamma$-Hilbert scheme of the surface $T^{*} E$.

A formula for the dimension of $\mathcal{M}_{\mathrm{Dol}}^{\mathrm{s}}\left(\hat{X}, n, \lambda_{\bullet}\right)$ is given in [14, p. 3], assuming the moduli space is nonempty:

$$
2(g-1) n^{2}+2+\sum_{p \in D}\left(n^{2}-\sum_{i=1}^{n_{p}}\left(\lambda_{p i+1}-\lambda_{p i}\right)^{2}\right)
$$

We have the estimate

$$
\sum_{i=1}^{n_{p}}\left(\lambda_{p i+1}-\lambda_{p i}\right)^{2} \leq n^{2}
$$

which follows from the inequality

$$
\sum_{i=1}^{n} x_{i}^{2} \leq\left(\sum_{i=1}^{n} x_{i}\right)^{2}
$$

where $x_{i} \geq 0$. In particular, we see that there are two possible cases, where expression (1) specializes to 2 . If $g=1$ and $D=0$, since inequality (2) is strict if there are two nonzero summands; and $g=0$ and

$$
-2 n^{2}+\sum_{p \in D}\left(n^{2}-\sum_{i=1}^{n_{p}-1}\left(\lambda_{p i+1}-\lambda_{p i}\right)^{2}\right)=0
$$


This expression on the other hand is $-2 q$, where $q$ denotes the quadratic form associated to the star-shaped graph

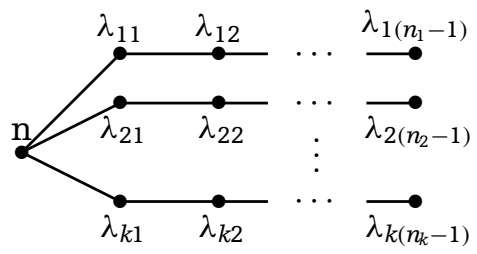

If $Q$ is of affine Dynkin type, we see in particular that all such dimension vectors are multiples of the basic imaginary root $\alpha$. In this paper, we only focus on the case where the dimension vector is equal to the basic imaginary root $\alpha$. The other cases are currently not accessible with our methods. We refer the reader however to the work of Kostov [32], which studies related varieties of local systems that arise in the study of the Deligne-Simpson problem.

\subsection{Duality for elliptic curves with symmetries}

Let $A$ be an abelian variety, the dual abelian variety $A^{\vee}$ is equivalent to the stack $\operatorname{Map}_{\text {grp }}\left(A, B \mathbb{G}_{m}\right)$ representing morphisms of group stacks $A \rightarrow B \mathbb{G}_{m}[49$, p. 184]. Equivalently, we can say that $A^{\vee}$ classifies extensions of $A$ by $\mathbb{G}_{m}$. This construction is analogous to the dual of a vector space $V^{\vee}:=\operatorname{Hom}(V, k)$, with $B \mathbb{G}_{m}$ taking the place of the 1D vector space $k$. For the same reason as there is a canonical morphism $V \rightarrow V^{\vee \vee}$ for vector spaces there is a canonical morphism

$$
\psi_{A}: A \rightarrow A^{\vee \vee},
$$

which is an isomorphism. This in turn gives rise to a morphism

$$
A \times A^{\vee} \rightarrow B \mathbb{G}_{m} .
$$

As the stack $B \mathbb{G}_{m}$ classifies line bundles, we see that there is a canonical line bundle $\mathcal{P}$ on $A \times A^{\vee}$, called Poincaré bundle. There is a general duality theory for group stacks, an exposition of which is given in [2].

It has been shown by Mukai [38] that the Poincaré line bundle $\mathcal{P}$ induces a natural equivalence of categories

$$
D_{\mathrm{coh}}^{b}(A) \cong D_{\mathrm{coh}}^{b}\left(A^{\vee}\right)
$$


If $\phi: A \rightarrow B$ is a morphism, we obtain a dual morphism $\phi^{\vee}: B^{\vee} \rightarrow A^{\vee}$ which sends an $S$-point $f: B \times S \rightarrow B \mathbb{G}_{m} \times S$ of $B^{\vee}$ to the composition

$$
A \times S \stackrel{\phi \times \operatorname{id}_{S}}{\longrightarrow} B \times S \stackrel{f}{\longrightarrow} B \mathbb{G}_{m} \times S .
$$

By definition the diagram

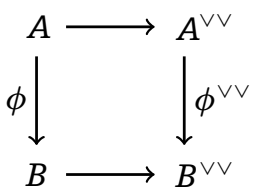

is commutative. In particular, we conclude that if $\Gamma$ is a finite group acting on $A$, then Mukai's equivalence (3) is $\Gamma$-equivariant; in the strong sense that the integral kernel $\mathcal{P}$ is endowed with a $\Gamma$-equivariant structure.

If $E$ denotes an elliptic curve, we may identify

$$
T^{*} E=E \times \mathcal{A}=\mathcal{M}_{\mathrm{Dol}}\left(E^{\vee}, 1\right),
$$

where $\mathcal{A}$ denotes the Hitchin base. Note that there is a canonical identification of elliptic curves $E \cong E^{\vee}$, given by the Abel-Jacobi map. This induces an identification of Hitchin bases

$$
\mathcal{A}(E)=\mathcal{A}\left(E^{\vee}\right)
$$

Using this autoequivalence, the remarks above, and Lemmas 3.5 and 3.8, we arrive at the following well-known observation.

Proposition 4.2. There is a canonical equivalence of derived categories of FourierMukai type relative to $\mathcal{A}$

$$
D_{\mathrm{coh}}^{b}\left(T^{*} E\right) \cong D_{\mathrm{coh}}^{b}\left(T^{*} E^{\vee}\right)
$$

If $E$ is equipped with a $\Gamma$-action, this equivalence respects the $\Gamma$-action, and in particular, we have an equivalence of derived categories of Fourier-Mukai type relative to $\mathcal{A} / \Gamma$

$$
D_{\mathrm{coh}}^{b}\left(\left[T^{*} E / \Gamma\right]\right) \cong D_{\mathrm{coh}}^{b}\left(\left[T^{*} E^{\vee} / \Gamma\right]\right) .
$$




\subsection{Higgs bundles and crepant resolutions}

In this subsection, we show how moduli spaces of Higgs bundles give rise to crepant resolutions of certain quotients of cotangent bundles of elliptic curves. We start with a technical definition, which turns out to be essential for relating Higgs bundles with torsion sheaves via Fourier-Mukai transform.

Definition 4.3. Let $X$ be an orbicurve and $(E, \theta)$ be a Higgs bundle on it. A composition series for $(E, \theta)$ is an increasing filtration by Higgs subbundles

$$
\left(E^{\bullet}, \theta\right) \subset(E, \theta)
$$

such that the successive quotients $E^{i+1} / E^{i}$, called factors, are locally free and have no nontrivial Higgs bundle as a quotient. The Higgs bundle $(E, \theta)$ is said to be admissible if there exists a composition series, such that all factors are of rank 1 and degree 0 . An $S$-family of Higgs bundles is called admissible if it is admissible over every geometric point of $S$. We denote the stack of rank $n$ admissible Higgs bundles on an orbicurve $X$ by $\mathcal{M}_{\mathrm{Dol}}^{\mathrm{ad}}(X, n)$.

It is a well-known fact [46, Lemma 4.2(1)] that an extension of semistable objects of the same slope is again semistable. We record the following implication for admissible Higgs bundles for later use.

Remark 4.4. Admissible Higgs bundles are semistable of slope zero.

We will see later that admissible Higgs bundles correspond to torsion sheaves supported on a dual Hitchin fibration. This will induce an equivalence of stacks.

Definition 4.5. For an orbisurface $S$, we denote by $\mathcal{T}(S, n)$ the stack of length $n$ torsion sheaves on $S$, that is, the 2-functor

$$
\operatorname{Aff}^{\text {op }} \rightarrow \text { Grpd }
$$

which sends an affine scheme $T$ to the groupoid of quasi-coherent sheaves $\mathcal{F}$ on $S \times T$, such that $\pi: \operatorname{supp} \mathcal{F} \rightarrow T$ is finite and $\pi_{*} \mathcal{F}$ is locally free of rank $n$ on $S$.

In the next lemma, we formulate how admissible Higgs bundles are related to torsion sheaves. 
Lemma 4.6. The equivalence of Proposition 4.2 gives rise to an equivalence of stacks

$$
\mathcal{T}\left(T^{*} E, n\right) \cong \mathcal{M}_{\mathrm{Dol}}^{\mathrm{ad}}\left(E^{\vee}, n\right)
$$

Proof. Given a $T$-point $\mathcal{F}$ of $\mathcal{T}\left(T^{*} E, n\right)$, we have to verify that the Fourier-Mukai transform $\Phi(\mathcal{F})$ on $T^{*} E^{\vee}$ is a $T$-family of quasi-coherent sheaves on $T^{*} E^{\vee}$, that is, a Higgs bundle on $E^{\vee}$ via the BNR correspondence (Proposition 2.2). This formulation is justified, as we know from Lemma 3.6 that for every $k$-scheme $T$ there is an induced Fourier-Mukai transform

$$
\Phi: D_{\mathrm{coh}}^{b}\left(T^{*} E \times T\right) \cong D_{\mathrm{coh}}^{b}\left(T^{*} E^{\vee} \times T\right)
$$

If

$$
\pi: T^{*} E^{\vee} \rightarrow E^{\vee}
$$

denotes the canonical projection, we need to verify that $\pi_{*} \Phi(\mathcal{F})$ is a locally free sheaf of rank $n$. This push-forward can be calculated as the Fourier-Mukai transform of $\mathcal{F}$ along the functor

$$
\Psi: D_{\mathrm{coh}}^{b}\left(T^{*} E \times T\right) \rightarrow D_{\mathrm{coh}}^{b}\left(E^{\vee} \times T\right)
$$

induced by the Poincaré bundle $\mathcal{P}$ on $E \times E^{\vee}$. Let $p_{1}: T^{*} E \times E^{\vee} \rightarrow T^{*} E$ and $p_{2}: T^{*} E \times$ $E^{\vee} \rightarrow E^{\vee}$ denote the canonical projections, respectively, their base changes with respect to $T$. Then we have

$$
\pi_{*} \Phi(\mathcal{F})=\Psi(F)=p_{2, *}\left(p_{1}^{*} \mathcal{F} \otimes \mathcal{P}\right)
$$

But since $\operatorname{supp} \mathcal{F} \rightarrow T$ is finite and $\mathcal{P}$ is a line bundle, we see that this is a locally free sheaf of rank $n$ on $E^{\vee}$. A similar Fourier-Mukai setup was used in [23] to define the vector bundle underlying the Higgs bundle constructed from a torsion sheaf.

We also need to check that the Fourier-Mukai transform $\Phi(\mathcal{F})$ is a family of admissible Higgs bundles on $[E / \Gamma]$. For this, we may replace $S$ by a geometric point and therefore assume that $\mathcal{F}$ has a composition series $\mathcal{F}^{\bullet}$, such that the successive quotients $\mathcal{F}^{i+1} / \mathcal{F}^{i}$ are skyscraper sheaves of length 1 . This composition series can be encoded in a sequence of distinguished triangles

$$
\mathcal{F}^{i} \longrightarrow \mathcal{F}^{i+1} \longrightarrow \mathcal{F}^{i+1} / \mathcal{F}^{i} \stackrel{\bullet}{\longrightarrow}
$$

Applying the equivalence $\Phi$ to $\mathcal{F}$, we see that $\Phi(\mathcal{F})$ may be filtered by distinguished triangles

$$
\Phi\left(\mathcal{F}^{i}\right) \rightarrow \Phi\left(\mathcal{F}^{i+1}\right) \rightarrow \Phi\left(\mathcal{F}^{i+1} / \mathcal{F}^{i}\right) \stackrel{\bullet}{\rightarrow}
$$


By assumption $\Phi\left(\mathcal{F}^{i+1} / \mathcal{F}^{i}\right)$ is a quasi-coherent sheaf $T^{*} E^{\vee}$, corresponding to a rank 1 degree 0 Higgs bundle on $E^{\vee}$ via the BNR correspondence (Proposition 2.2). By induction on $n$, we obtain that $\Phi(\mathcal{F})$ corresponds to an admissible Higgs bundle.

Similarly, we see that an admissible Higgs bundle of rank $n$ on $E^{\vee}$ is sent to a length $n$ torsion-free sheaf on $T^{*} E$.

We have found a way of relating torsion sheaves on the surface $T^{*} E$ to Higgs bundles on the dual elliptic curve $E^{\vee}$. As a next step, we investigate the transform of a point of the $\Gamma$-Hilbert scheme $Y$ of $T^{*} E$. Such a point gives rise to a $\Gamma$-equivariant torsion sheaf $\mathcal{F}$ on $T^{*} E$ together with a $\Gamma$-equivariant surjection $s: \mathcal{O}_{T^{*} E} \rightarrow \mathcal{F}$. As a first approximation, we expect to obtain a $\Gamma$-equivariant Higgs bundle of $\operatorname{rank}|\Gamma|$ on $E^{\vee}$, due to the functoriality of the construction described above. In the proof below, we investigate the structure corresponding to the surjection $s$.

Proof of Theorem 4.1. The $\Gamma$-Hilbert scheme $Y$ of $T^{*} E$ can be defined in terms of $\mathcal{T}=$ $\mathcal{T}\left(T^{*}[E / \Gamma]\right)$. An $S$-point of $Y$ consists of an $S$-point $\mathcal{F}$ of $\mathcal{T}$ together with a surjection

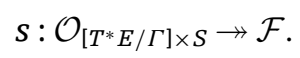

Moreover, we demand that the $\Gamma$-representation

$$
\operatorname{Hom}\left(\mathcal{O}_{\left[T^{*} E / \Gamma\right] \times S}, \mathcal{F}\right)
$$

is the regular $S$-linear representation of $\Gamma$. We can now try to understand how $s$ transforms under the equivalence of categories $\Phi$.

Let us denote by $T_{0}^{*} E$ the closed subscheme of $T^{*} E$ given by the fiber over zero of $T^{*} E \rightarrow E$. The equivalence $D_{\text {coh }}^{b}\left(T^{*} E\right) \cong D_{\text {coh }}^{b}\left(T^{*} E^{\vee}\right)$ sends $\mathcal{O}_{T^{*} E}$ to $\mathcal{O}_{T_{0}^{*} E^{\vee}}$ [-1]. In particular, we see that $v:=\Phi(s)$ is a morphism

$$
v: \mathcal{O}_{T_{0}^{*} E^{\vee}}[-1] \rightarrow \Phi(\mathcal{F})
$$

Serre duality tells us that this is equivalent to a morphism

$$
v^{\prime}:\left(\Phi(\mathcal{F})_{0}\right)^{\vee} \rightarrow k,
$$


that is, an element $v \in \Phi(\mathcal{F})_{0}$. Under this equivalence, a morphism $\mathcal{O}_{T^{*} E} \rightarrow \mathcal{F}$ corresponds to a linear map $\tau_{*}\left(V^{\vee} \otimes \mathcal{O} / L_{0}^{-1}\right) \rightarrow k$, where $(V, \theta)$ is the $\Gamma$-equivariant Higgs bundle associated to $\mathcal{F} \in \mathcal{T}$, and we use the notation of Definition 2.15. But

$$
\tau_{*}\left(V^{\vee} \otimes \mathcal{O} / L_{0}^{-1}\right) \cong E_{0}^{\vee} / F_{1}^{\vee},
$$

where we use the notation of Definition 2.4 to denote the quasi-parabolic structure. In particular, we obtain a nontrivial linear map

$$
k \rightarrow F_{n_{0}-1},
$$

by dualizing, that is, a nonzero vector $v \in F_{n_{0}-1}$.

The vector space $\operatorname{Hom}\left(\mathcal{O}_{\left[T^{*} E / \Gamma\right] \times S}, \mathcal{F}\right)$ corresponds to $\Phi(\mathcal{F})_{0}$, as the argument given above tell us. In particular, we see that $Y$ is equivalent to the moduli stack of the data

$$
(\mathcal{E}, \theta, v)
$$

where $(\mathcal{E}, \theta)$ is an admissible Higgs bundle on $\left[T^{*} E / \Gamma\right], \mathcal{E}_{0}$ is the regular $\Gamma$ representation, and $v \in \mathcal{E}_{0}^{\Gamma}$ is a nonzero vector spanning the invariant part of $\mathcal{E}_{0}$. The latter is naturally equivalent to the moduli space of admissible Higgs bundles on $\left[T^{*} E / \Gamma\right]$, such that $\mathcal{E}_{0}$ carries the regular representation. Now we may apply Lemma 2.20 to see that this corresponds exactly to the required type of parabolic bundles.

Stability of the parabolic Higgs bundles follows from the fact that all $\Gamma$ invariant subbundles are of orbifold slope $\leq 0$ (see Remark 4.4) and that $\Phi(\mathcal{F})$ is the only degree 0 subbundle containing $v$. Since the weights are the canonical weights except from $\alpha_{n-1}$, stability follows.

Note that $Y$ is naturally a $\mathcal{A} / \Gamma$-space with respect to the structural morphism

$$
Y \rightarrow T^{*} E / \Gamma \rightarrow \mathcal{A} / \Gamma
$$

Here the first morphism is the Hilbert-Chow morphism. The fact that $\Phi: D_{\operatorname{coh}}^{b}\left(\left[T^{*} E / \Gamma\right]\right) \cong$ $D_{\text {coh }}^{b}\left(\left[T^{*} E^{\vee} / \Gamma\right]\right.$ is defined relative to $\mathcal{A} / \Gamma$ implies that the morphism $Y \rightarrow \mathcal{M}\left(\left[\widehat{E^{\vee} / \Gamma}\right], Q, \lambda\right)$ is a morphism of $\mathcal{A} / \Gamma$-spaces. We observe as well that this map is proper.

Therefore, we have a morphism

$$
Y \rightarrow \mathcal{M}\left(\left[\widehat{E^{\vee} / \Gamma}\right], Q, \lambda\right)
$$

of proper $\mathcal{A} / \Gamma$-spaces. Since both spaces are of equal dimension and connected, we conclude that it is surjective. In particular, we obtain that every Higgs bundle in the 
moduli space $\mathcal{M}\left(\left[\widehat{E^{\vee} / \Gamma}\right], Q, \lambda\right)$ is admissible. This allows us to conclude that

$$
Y \cong \mathcal{M}\left(\left[\widehat{E^{\vee} / \Gamma}\right], Q, \lambda\right)
$$

with the inverse map provided by the inverse of the Fourier-Mukai transform $\Phi^{-1}$.

\subsection{Local systems and crepant resolutions}

Using the categorification of geometric class field theory obtained in [34,48], we are able to prove the analogous result for moduli spaces of local systems by similar techniques. In the following, we denote by $D_{\mathrm{qcoh}}\left(X, D_{X}\right)$ the derived category of quasi-coherent $D_{X^{-}}$ modules on a smooth variety $X$.

Theorem 4.7 (Laumon and Rothstein). If $A$ is an abelian variety defined over an algebraically closed field of characteristic zero, we denote by $A^{\sharp}$ the moduli space of rank 1 local systems on $A$. Then there exists a canonical equivalence of derived categories

$$
\Phi_{C F T}: D_{\mathrm{qcoh}}\left(A^{\sharp}\right) \cong D_{\mathrm{qcoh}}\left(A^{\vee}, D_{A^{\vee}}\right) .
$$

Note that in positive characteristic we define the ring of differential operators $D_{X}$ of a smooth variety to be the universal enveloping algebra of the Lie algebroid of tangent vectors $\Theta_{X}$. The analog of the above theorem in positive characteristic is proved in [19, Corollary 3.8], using the techniques developed in [9, 10].

Theorem 4.8 (Chen-Zhu). If $A$ is an abelian variety defined over an algebraically closed field of positive characteristic, we denote by $A^{\sharp}$ the moduli space of rank 1 local systems on $A$. Then there is a canonical equivalence of derived categories

$$
D_{\mathrm{qcoh}}\left(A^{\sharp}\right) \cong D_{\mathrm{qcoh}}\left(A^{\vee}, D_{A^{\vee}}\right) .
$$

Remark 4.9. In this paper, we will only be interested in the case where $A=E$ is an elliptic curve. This special case is also covered by [9, Theorem 4.10(2)].

As before, we start by relating torsion sheaves on the surface $\mathcal{M}_{\mathrm{dR}}(E, 1)=E^{\sharp}$ with local systems on $E^{\vee}$. Although the next proposition is completely analogous to Lemma 4.6, it is more powerful, since every local system defined over an algebraically closed field of characteristic zero is admissible due to the fact that every vector bundle on a curve supporting an algebraic connection has degree 0. As in Definition 4.3, one defines admissible local systems. We observe, however, that in characteristic zero every 
local system is automatically admissible, since a vector bundle carrying an algebraic flat connection has to be of degree 0 .

Proposition 4.10. The equivalence $\Phi_{C F T}$ of Theorem 4.7 (respectively, Theorem 4.8) induces an equivalence of stacks

$$
\mathcal{T}\left(E^{\sharp}, n\right) \cong \mathcal{M}_{\mathrm{dR}}^{\mathrm{ad}}\left(E^{\vee}, n\right),
$$

relating length $n$ torsion sheaves on the surface $E^{\sharp}$ to admissible rank $n$ local systems on $E^{\vee}$. If the base field $k$ is of zero characteristic, then $\mathcal{M}_{\mathrm{dR}}^{\mathrm{ad}}\left(E^{\vee}, n\right)=\mathcal{M}_{\mathrm{dR}}\left(E^{\vee}, n\right)$.

Proof. Let us denote by $S$ an affine scheme, by $X$ an arbitrary smooth scheme, and by $D_{\mathrm{qcoh}}\left(X \times S, p_{X}^{*} D_{X}\right)$ the derived category of $p_{X}^{*} D_{X}$-modules, where $p_{X}: X \times S \rightarrow X$ is the canonical projection. Objects of this category should be thought of as $S$-families of complexes of $D_{X}$-modules. It is clear that we also have an equivalence of derived categories

$$
D_{\mathrm{qcoh}}\left(A^{\sharp} \times S\right) \cong D_{\mathrm{qcoh}}\left(A^{\vee} \times S, p_{A^{\vee}}^{*} D_{A^{\vee}}\right)
$$

as it follows, for instance, from [8, Proposition 4.1] and the fact that the above equivalence of Laumon and Rothstein can be lifted to the canonical enhancements as stable $\infty$-categories.

Using the forgetful functor

$$
\Psi: D_{\mathrm{qcoh}}\left(E^{\vee}, D_{E^{\vee}}\right) \rightarrow D_{\mathrm{qcoh}}\left(E^{\vee}\right)
$$

we can describe the underlying quasi-coherent sheaf $\left(\Psi \circ \Phi_{C F T}\right)(\mathcal{F})$ as the Fourier-Mukai transform

$$
D_{\mathrm{qcoh}}\left(E^{\sharp}\right) \rightarrow D_{\mathrm{qcoh}}\left(E^{\vee}\right),
$$

with integral kernel given by the universal flat connection $\mathcal{L}$ on $E^{\sharp} \times E$. As in the proof of Lemma 4.6, we obtain therefore that $\Phi_{C F T}(\mathcal{F})$ is a complex of a family of $D$-modules concentrated in a single degree.

Vice versa starting with a family of local systems $(V, \nabla)$ on $E^{\vee}$, we see from the existence of a composition series for $(V, \nabla)$ as in the proof of lemma 4.6 that $\Phi_{C F T}^{-1}(V)$ is a torsion sheaf on $E^{\sharp}$.

For $k=\mathbb{C}$, Proposition 4.10 seems natural from a complex analytic viewpoint. Since $\pi_{1}(E) \cong \mathbb{Z}^{2}$, the Riemann-Hilbert correspondence implies that $\mathcal{M}_{\mathrm{dR}}(E, n)$ is 
complex analytically isomorphic to the quotient stack

$$
\left[\left\{(A, B) \in \mathrm{GL}_{n} \times \mathrm{GL}_{n} \mid A B=B A\right\} / \mathrm{GL}_{n}\right] .
$$

This algebraic quotient stack in turn is equivalent to $\mathcal{T}\left(\mathbb{C}^{\times} \times \mathbb{C}^{\times}, n\right)$, as we record below.

Remark 4.11. Let $k$ be an algebraically closed field. There exists a canonical equivalence of stacks

$$
\mathcal{T}\left(\mathbb{G}_{m}^{r} \times \mathbb{A}^{s}, n\right) \cong\left[\left\{\left(A_{1}, \ldots, A_{r}, A_{r+1}, \ldots, A_{r+s}\right) \in \mathrm{GL}_{n}^{r} \times \mathfrak{g l}_{n}^{s} \mid\left[A_{i}, A_{j}\right]=0 \forall(i, j)\right\} / \mathrm{GL}_{n}\right],
$$

where $\mathrm{GL}_{n}$ acts by conjugation on this variety of matrices.

Proof. The data of a length $n$ torsion sheaf on $\mathbb{G}_{m}^{r} \times \mathbb{A}^{s}$ are equivalent to a rank $n$ $k$-vector space $V$, endowed with the structure of a $k\left[X_{1}^{ \pm 1}, \ldots, X_{r}^{ \pm 1}, X_{r+1}, \ldots, X_{r+s}\right]$-module. This in turn is tautologically the same thing as a $k$-vector space $V$ together with $r+s$ pairwise commuting endomorphisms $\left(A_{i}\right)_{i=1, \ldots, r+s}$, such that $\operatorname{det} A_{i} \neq 0$ for $i \leq r$. As the same statements hold in families, we conclude the proof of the assertion.

On the other hand, the surface $\mathbb{C}^{\times} \times \mathbb{C}^{\times}$is complex analytically equivalent to $E^{\sharp}$, which induces an isomorphism of complex analytic stacks

$$
\mathcal{T}\left(E^{\sharp}, n\right) \cong \mathcal{T}\left(\mathbb{C}^{\times} \times \mathbb{C}^{\times}, n\right) \cong \mathcal{M}_{\mathrm{dR}}(E, n) .
$$

Proposition 4.10 allows us to prove a version of Theorem 4.1 for moduli spaces of parabolic local systems, by the exact same methods.

Theorem 4.12. Let $k$ be an algebraically closed field of characteristic zero or $p>|\Gamma|$. The moduli space of stable parabolic local systems $\mathcal{M}_{\mathrm{dR}}^{\mathrm{s}}(Q, \lambda)$ of orbifold degree 0 , associated to the orbifold $[E / \Gamma]$ with the same weights as in Theorem 4.1 and eigenvalues given by the canonical weights, is naturally isomorphic to the $\Gamma$-Hilbert scheme $\left(E^{\sharp}\right)^{[\Gamma]}$ of the surface $E^{\sharp}$.

Proof. As in the proof of Theorem 4.1, we use Proposition 4.10 and Serre duality to construct a morphism

$$
\left(E^{\sharp}\right)^{[\Gamma]} \rightarrow \mathcal{M}_{\mathrm{dR}}^{\mathrm{s}}(Q, \lambda) .
$$


In order to show that this is an isomorphism, it suffices to establish that every local system in $\mathcal{M}_{\mathrm{dR}}^{\mathrm{s}}(O, \lambda)$ is admissible. In characteristic zero, this is automatically satisfied, since every vector bundle admitting of an algebraic flat connection is of degree 0. In positive characteristic, one has a proper Hitchin map, at one's disposal, as explained in Section 5.3 and [24]. This allows us to conclude the proof as the one of Theorem 4.1.

In the $\tilde{D}_{4}$-case, similar 2D moduli spaces of flat connections have been studied by Okamoto [43] in the context of the sixth Painlevé equation.

\subsection{Derived equivalences}

In Proposition 4.2, we have shown that there is a derived equivalence

$$
D_{\mathrm{coh}}^{b}\left(\left[T^{*} E^{\vee} / \Gamma\right]\right) \cong D_{\mathrm{coh}}^{b}\left(\left[T^{*} E / \Gamma\right]\right)
$$

Using Theorem 4.1 and the derived equivalence of Theorem 3.9, we arrive at a string of equivalences

$$
D_{\mathrm{coh}}^{b}(\mathcal{M}) \cong D_{\mathrm{coh}}^{b}\left(\left[T^{*} E^{\vee} / \Gamma\right]\right) \cong D_{\mathrm{coh}}^{b}\left(\left[T^{*} E / \Gamma\right]\right) \cong D_{\mathrm{coh}}^{b}\left(\mathcal{M}^{\vee}\right),
$$

where $\mathcal{M}$ and $\mathcal{M}^{\vee}$ denote the respective moduli spaces of parabolic Higgs bundles.

Theorem 4.13. Let $\mathcal{M}:=\mathcal{M}_{\mathrm{Dol}}(\widehat{[E / \Gamma]}, Q)$ denote the moduli space over the Hitchin base $\mathcal{A}$ studied in Section 4.2. We have a natural equivalence of derived categories of FourierMukai type

$$
\Phi: D_{\mathrm{coh}}^{b}(\mathcal{M}) \cong D_{\mathrm{coh}}^{b}\left(\mathcal{M}^{\vee}\right)
$$

relative to $\mathcal{A}$, extending the Fourier-Mukai transform for dual abelian varieties over the locus $\mathcal{A}^{\text {sm }}$. The corresponding Fourier-Mukai kernel is given by a Cohen-Macaulay sheaf $\overline{\mathcal{P}}$ on the fiber product $\mathcal{M} \times{ }_{\mathcal{A}} \mathcal{M}^{\vee}$.

Proof. This is an equivalence of Fourier-Mukai type relative to $\mathcal{A}$ by construction. Therefore, we only need to verify the second assertion, namely that the integral kernel $\overline{\mathcal{P}}$ restricts to the Fourier-Mukai transform associated to the Poincaré bundle $\mathcal{P}$ on

$$
\mathcal{M}^{\mathrm{sm}} \times{ }_{\mathcal{A}^{\mathrm{sm}}} \mathcal{M}^{\vee, \mathrm{sm}} .
$$


Over the smooth locus $\mathcal{A}^{\text {sm }}$ the two morphisms

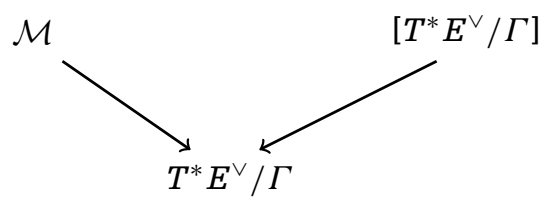

are actually isomorphisms and the restriction of the equivalence of Theorem 3.9 to the smooth locus (which is possible because of Lemma 3.10) is the equivalence induced from this isomorphism. Étale locally on $\mathcal{A}^{\mathrm{sm}}$ we may identify the relative abelian variety given by the Hitchin fibration with $E^{\vee}$. We see that the equivalence in question is just FourierMukai duality for the abelian variety $E$.

To verify the last assertion, we need to show that the equivalence $D_{\text {coh }}^{b}\left(\mathcal{M}^{\vee}\right) \cong$ $D_{\text {coh }}^{b}(\mathcal{M})$ sends the $\mathcal{M}^{\vee}$-family of quasi-coherent sheaves on $\mathcal{M}^{\vee}$ given by the structure sheaf of the diagonal $\Delta_{*} \mathcal{O}_{\mathcal{M}}$ to a Cohen-Macaulay sheaf. This equivalence can be divided into several steps

$$
D_{\mathrm{coh}}^{b}(\mathcal{M}) \cong D_{\mathrm{coh}}^{b}\left(\left[T^{*} E^{\vee} / \Gamma\right]\right) \cong D_{\mathrm{coh}}^{b}\left(\left[T^{*} E / \Gamma\right]\right) \cong D_{\mathrm{coh}}^{b}\left(\mathcal{M}^{\vee}\right)
$$

According to Theorem 4.1, the composition of the first two equivalences send $\Delta_{*} \mathcal{O}_{\mathcal{M}^{\vee}}$ to the universal family $\overline{\mathcal{Q}}$ of Higgs orbibundles on $\mathcal{M}^{\vee} \times\left[T^{*} E / \Gamma\right]$. If

$$
\pi:\left[T^{*} E / \Gamma\right] \rightarrow[E / \Gamma]
$$

denotes the canonical projection, we have that

$$
\left(\operatorname{id}_{\mathcal{M}} \times \pi\right)_{*} \overline{\mathcal{Q}},
$$

the $\mathcal{M}$-family of vector bundles underlying the universal family of Higgs bundles $\overline{\mathcal{Q}}$. In particular, since $\pi: \operatorname{supp} \overline{\mathcal{Q}} \rightarrow[E / \Gamma]$ is finite, we see that $\overline{\mathcal{Q}}$ is Cohen-Macaulay. Therefore, we need to show that the equivalence $\Psi: D_{\mathrm{coh}}^{b}\left(\left[T^{*} E / \Gamma\right]\right) \cong D_{\mathrm{coh}}^{b}\left(\mathcal{M}^{\vee}\right)$ sends $\overline{\mathcal{Q}}$ to a Cohen-Macaulay sheaf $\overline{\mathcal{P}}$ on $\mathcal{M} \times_{\mathcal{A}} \mathcal{M}^{\vee}$.

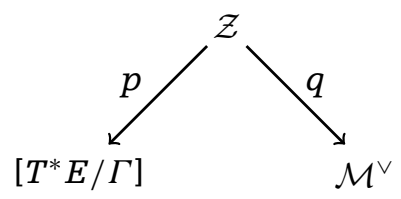

The universal $\Gamma$-cluster is endowed with a line bundle $\mathcal{K}$ and $\Psi$ can be written as

$$
R q_{*}\left(L p^{*}-\otimes^{L} \mathcal{K}\right)
$$


Because $q$ is a finite morphism and $\mathcal{K}$ is a line bundle,

$$
\Psi(\overline{\mathcal{Q}})=R(\mathrm{id} \times q)_{*}\left(L(\mathrm{id} \times p)^{*} \overline{\mathcal{Q}} \otimes^{L} \mathcal{K}\right)
$$

is Cohen-Macaulay if and only if $L p^{*} \overline{\mathcal{Q}}$ is Cohen-Macaulay. Lemma 2.3 of [3] implies Cohen-Macaulyness of this pullback, if $\mathcal{M} \times{ }_{\mathcal{A}}\left[T^{*} E / \Gamma\right]$ is Gorenstein, $(\mathrm{id} \times p)$ is Torfinite and $\mathcal{M} \times{ }_{\mathcal{A}} \mathcal{Z}$ is Cohen-Macaulay. Tor-finiteness of $p$ follows from smoothness of $\mathcal{M}$ and is preserved by base change along a flat morphism. The two fiber products $\mathcal{M} \times{ }_{\mathcal{A}}\left[T^{*} E / \Gamma\right]$ and $\mathcal{M} \times{ }_{\mathcal{A}} \mathcal{M}$ are locally complete intersections (see tags $01 \mathrm{UH}, 01 \mathrm{UI}$ in [55]), and

$$
\mathcal{M} \times_{\mathcal{A}} \mathcal{Z} \rightarrow \mathcal{M} \times_{\mathcal{A}} \mathcal{M}
$$

is a finite morphism, which implies Cohen-Macaulayness of $\mathcal{M} \times_{\mathcal{A}} \mathcal{Z}$.

We obtain a similar result for moduli spaces of flat connections, which should be seen as an instance of the Geometric Langlands correspondence.

Theorem 4.14. Let $\mathcal{M}_{\mathrm{dR}}^{\mathrm{s}}(\widehat{[E / \Gamma]}, Q, \lambda)$ denote the moduli space of local systems studied in Section 4.2. We have a natural equivalence of derived categories

$$
\Phi_{G L}: D_{\mathrm{qcoh}}\left(\mathcal{M}_{\mathrm{dR}}^{\mathrm{s}}(\widehat{[E / \Gamma]}, Q, \lambda)\right) \cong D_{\mathrm{qcoh}}\left(\left[E^{\vee} / \Gamma\right], D_{\left[E^{\vee} / \Gamma\right]}\right)
$$

Proof. For a smooth stack $\mathcal{Y}$, let us denote by $\mathcal{D}(Y)$ the canonical $\infty$-enhancement of the derived category of $D$-modules on $\mathcal{Y}$, to which we have already alluded to in the proof of Proposition 4.10. According to Theorem 4.7, we have an equivalence

$$
O C\left(E^{\sharp}\right) \cong \mathcal{D}\left(E^{\vee}\right) .
$$

Taking $\Gamma$-equivariant objects, that is, utilizing the same construction as in the proof of Lemma 3.3, we recover the above equivalence.

\section{Hilbert Schemes, Higgs Bundles, and Local Systems}

If $Q$ is a graph with a marked vertex $v$, we denote by $Q^{\prime}$ the quiver obtained by adjoining an extra edge, linking $v$ with a new vertex $v^{\prime}$. If $\lambda$ is a dimension vector for $Q$, we denote by $\lambda^{\prime}$ the dimension vector satisfying

$$
\lambda^{\prime} \mid Q=\lambda,
$$

and $\lambda^{\prime}\left(v^{\prime}\right)=1$. 
If $O$ is a Dynkin diagram, then the associated affine Dynkin diagram $\tilde{Q}$ has a marked vertex $v$, called the affine vertex. In this section, we discuss the geometric analog for moduli spaces of Higgs bundles and local systems of the transition

$$
\tilde{Q} \rightsquigarrow \tilde{Q}^{\prime} .
$$

\subsection{Hilbert schemes as moduli spaces}

Theorem 5.1. Let $k$ be an algebraically closed field of characteristic zero or $p>$ $\max (|\Gamma|, n)$. We denote by $\mathcal{M}$ the moduli spaces of parabolic Higgs bundles $\mathcal{M}_{\mathrm{Dol}}^{\mathrm{s}}(Q, \lambda)$ from Theorem 4.1. Then the Hilbert scheme $\mathcal{M}^{[n]}$ is again a moduli space of Higgs bundles. More precisely, we have

$$
\mathcal{M}^{[n]} \cong \mathcal{M}_{\mathrm{Dol}}^{\mathrm{s}}\left(\tilde{Q}^{\prime},(n \lambda)^{\prime}\right)
$$

where the weights at the marked point corresponding to $0 \in E$ are $\alpha_{i}:=\frac{i}{n}$ for $i<n$ and $1>$ $\alpha_{n}>\frac{n-1}{n}$, and all the other weights are given by the canonical weights; and the orbifold degree is 0 . The Hitchin map $\mathcal{M}^{[n]} \rightarrow \mathcal{A}_{n}$ factors through the Hilbert-Chow map

$$
\mathcal{M}^{[n]} \rightarrow \mathcal{M}^{(n)} \rightarrow \mathcal{A}_{1}^{(n)}=\mathcal{A}_{n}
$$

where $\mathcal{M}^{(n)} \rightarrow \mathcal{A}_{1}^{(n)}$ is the map induced by $\mathcal{M}^{n} \rightarrow \mathcal{A}_{1}^{n}$.

In the case of $Q=\tilde{A}_{0}$, this is a theorem of Gorsky-Nekrasov-Rubtsov [23, Section 5.1].

Proof. Theorems 3.9 and 4.13 imply that we have an equivalence

$$
D_{\mathrm{coh}}^{b}(\mathcal{M}) \cong D_{\mathrm{coh}}^{b}\left(\left[T^{*} E / \Gamma\right]\right)
$$

defined relative to $\mathcal{A}_{1}$. In particular, we can show as in Lemma 4.6 that the moduli stack of length $n$ torsion sheaves on $\mathcal{M}$ is equivalent to the moduli stack of $\Gamma$-equivariant rank $n$ admissible Higgs bundles on $E$ :

$$
\mathcal{T}(\mathcal{M}) \cong \mathcal{M}_{\mathrm{Dol}}([E / \Gamma], n) .
$$

As in the proof of Theorem 4.1, we see that under this equivalence a morphism $\mathcal{O}_{\mathcal{M}} \rightarrow \mathcal{F}$ corresponds to a linear map $\tau_{*}\left(V^{\vee} \otimes \mathcal{O} / L_{0}^{-1}\right) \rightarrow k$, where $(V, \theta)$ is the $\Gamma$-equivariant Higgs 
bundle associated to $\mathcal{F} \in \mathcal{T}$. But

$$
\tau_{*}\left(V^{\vee} \otimes \mathcal{O} / L_{0}^{-1}\right) \cong E_{0}^{\vee} / F_{1}^{\vee}
$$

In particular, we obtain a nontrivial linear map

$$
k \rightarrow F_{n_{0}-1}
$$

by dualizing, that is, a nonzero vector $v \in F_{n_{0}-1}$.

We claim that the condition that $\mathcal{O}_{\mathcal{M}} \rightarrow \mathcal{F}$ is surjective, equivalent to the fact that $(V, \theta, v)$ does not contain any degree 0 Higgs subbundles containing $v$.

Let us assume that $\mathcal{O}_{\mathcal{M}}$ is surjective. If $(V, \theta, v)$ contains a nontrivial degree 0 Higgs subbundle, which contains $v$, then there is a smallest such Higgs subbundle $\left(V^{\prime}, \theta, v\right)$ of rank $k<n$. In particular, its transform $\mathcal{G}$ gives rise to a commutative diagram

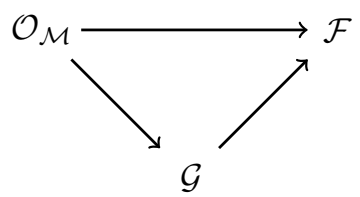

Because the horizontal arrow is surjective and $W$ is a length $k$ torsion sheaf, this is a contradiction.

Similarly, one shows that if $(V, \theta, v)$ does not contain a nontrivial degree 0 Higgs subbundle containing $v$, then the corresponding morphism $\mathcal{O}_{\mathcal{M}} \rightarrow \mathcal{F}$ is surjective. Namely, if it is not surjective, its image gives rise to a nontrivial Higgs subbundle of $(V, \theta, v)$ containing $v$. Stability is checked as in the proof of Theorem 4.1.

We obtain a morphism of $\mathcal{A}_{n}$-spaces

$$
\mathcal{M}^{[n]} \rightarrow \mathcal{M}_{\mathrm{Dol}}^{\mathrm{s}}\left(\tilde{Q}^{\prime},(n \lambda)^{\prime}\right)
$$

as the type of the corresponding parabolic bundle can be checked for a single point, by connectivity of the moduli spaces, for instance over the locus of smooth spectral curves. Properness of the Hitchin morphism and the fact that both spaces have equal dimension and are connected, imply that this morphism is surjective. In particular, we may conclude that every parabolic Higgs bundle in $\mathcal{M}_{\mathrm{Dol}}^{\mathrm{s}}\left(\tilde{Q}^{\prime},(n \lambda)^{\prime}\right)$ is admissible. This implies that the above morphism is an isomorphism, with the inverse given by the inverse Fourier-Mukai transform.

There is an analogous statement for moduli spaces of local systems that is proved by the same means as the Theorems 4.12 and 5.1. The role of the derived 
equivalence $D_{\text {coh }}^{b}\left(T^{*} E\right) \cong D_{\text {con }}^{b}\left(T^{*} E^{\vee}\right)$ is taken by Laumon-Rothstein's geometric class field theory (Theorem 4.7).

Theorem 5.2. Let $k$ be an algebraically closed field of characteristic zero or $p>$ $\max (|\Gamma|, n)$. We denote by $\mathcal{M}$ one of the moduli spaces of parabolic local systems $\mathcal{M}_{\mathrm{dR}}^{\mathrm{s}}(O, \lambda)$ from Theorem 4.12 defined over $k$. Then the Hilbert scheme $\mathcal{M}^{[n]}$ is again a moduli space of local systems. More precisely, we have

$$
\mathcal{M}^{[n]} \cong \mathcal{M}_{\mathrm{dR}}^{\mathrm{s}}\left(\tilde{Q}^{\prime},(n \lambda)^{\prime}\right),
$$

where the weights $\alpha_{i}$ are as in Theorem 5.1, the orbifold degree is fixed to be 0 and the eigenvalues of the residues are given by $\omega_{i}:=\alpha_{i}$ for $i<n$ and $\omega_{n}:=\alpha_{n-1}$.

Remark 5.3. The reader might be wondering whether the moduli spaces of Theorems 5.1 and 5.2 are related via nonabelian Hodge theory [51]. According to [51, Table, p. 720], the weight $\alpha$ and eigenvalue $b+\mathrm{i} c$ of the residues of a parabolic Higgs bundle give rise to weight $\alpha-2 b$ and eigenvalue $\alpha+\mathrm{i} 2 c$ of the corresponding parabolic local system. In our case, the eigenvalues are zero in the Higgs case (nilpotent residue). In particular, we see that these Higgs bundles correspond to local systems with the same weights, but eigenvalues given by the weights. Comparing weights and eigenvalues in the local system case, as given by Theorem 5.2, one sees that the two moduli spaces do not correspond to each other with respect to nonabelian Hodge theory.

\subsection{Derived equivalences}

We begin this subsection with the following observation. We refer the reader to [44, Proposition 8], where this result is proved for varieties defined over the field of complex numbers.

Lemma 5.4. Let $X$ and $Y$ be two quasi-projective smooth surfaces defined over an algebraically closed field of characteristic $p>n$. If we have an equivalence of derived categories

$$
D_{\text {coh }}^{b}(X) \cong D_{\text {coh }}^{b}(Y)
$$

of Fourier-Mukai type, then this induces an equivalence

$$
D_{\text {coh }}^{b}\left(X^{[n]}\right) \cong D_{\text {coh }}^{b}\left(Y^{[n]}\right)
$$

of Fourier-Mukai type. 
Proof. Lemma 3.7 allows us to take the $n$th power of the equivalence $D_{\text {coh }}^{b}(X) \cong D_{\text {coh }}^{b}(Y)$, $D_{\mathrm{coh}}^{b}\left(X^{n}\right) \cong D_{\mathrm{coh}}^{b}\left(Y^{n}\right)$

On both spaces we have a natural action of the symmetric group $S_{n}$ by permuting the factors. The integral kernel is a sheaf naturally endowed with an $S_{n}$-equivariant structure; therefore, we may apply Lemma 3.8 and conclude that

$$
D_{\mathrm{coh}}^{b}\left(\left[X^{n} / S_{n}\right]\right) \cong D_{\mathrm{coh}}^{b}\left(\left[Y^{n} / S_{n}\right]\right)
$$

Together with Corollary 3.12, we obtain

$$
D_{\mathrm{coh}}^{b}\left(X^{[n]}\right) \cong D_{\mathrm{coh}}^{b}\left(Y^{[n]}\right)
$$

Theorem 5.5. We denote by $\mathcal{M}^{[n]}$ the moduli space of parabolic Higgs bundles associated to $[E / \Gamma]$ of Theorem 5.1. By $\mathcal{M}^{\vee[n]}$, we denote the same moduli space for $\left[\widehat{E^{\vee} / \Gamma}\right]$. Both moduli spaces $\mathcal{M}^{[n]}$ and $\mathcal{M}^{\vee[n]}$ are $\mathcal{A}$-spaces, where $\mathcal{A}$ is the Hitchin base. Under the assumptions of Lemma 5.4, there is a canonical equivalence of derived categories

$$
D_{\mathrm{coh}}^{b}\left(\mathcal{M}^{[n]}\right) \cong D_{\mathrm{coh}}^{b}\left(\mathcal{M}^{\vee[n]}\right)
$$

relative to $\mathcal{A}$. The integral kernel of this derived equivalence is a Cohen-Macaulay sheaf $\overline{\mathcal{P}}$ on $\mathcal{M}^{[n]} \times{ }_{\mathcal{A}} \mathcal{M}^{\vee[n]}$, which restricts to the Poincaré bundle $\mathcal{P}$ over the locus of smooth spectral curves $\mathcal{A}^{\mathrm{sm}}$.

Proof. This is a consequence of Theorem 5.1 and Lemma 5.4. Note that the construction in the proof of this lemma respects the morphism to the Hitchin base (due to Lemma 3.10). The last two assertions are verified as in the proof of Theorem 4.13, with the single exception that this time the sheaf $\overline{\mathcal{Q}}$ cannot be thought of as a universal family of Higgs bundles. Therefore, Cohen-Macaulayness has to be established by different means. The sheaf $\overline{\mathcal{Q}}$ is the transform of the structure sheaf $\mathcal{O}_{\mathcal{Z}}$ of the universal $S_{n}$-cluster on $\mathcal{M}^{[n]} \times_{\mathcal{A}}\left[M^{n} / S_{n}\right]$ along the equivalence $D_{\mathrm{qcoh}}^{b}\left(\left[M^{n} / S_{n}\right]\right) \cong D_{\mathrm{qcoh}}^{b}\left(\left[M^{\vee n} / S_{n}\right]\right)$; we denote the integral kernel of the latter equivalence by $\mathcal{R}$, and it is Cohen-Macaulay according to Theorem 4.13 and Lemma 3.7. Let $\iota: \mathcal{Z} \rightarrow\left[M^{n} / S_{n}\right]$ be the canonical morphism; [3, Lemma 2.3] implies that $L \iota^{*} \mathcal{R}$ is Cohen-Macaulay, since $\mathcal{Z}$ is finite over $\mathcal{M}^{[n]}$ and therefore Cohen-Macaulay itself. The natural morphism $\pi: \mathcal{Z} \rightarrow \mathcal{M}^{[n]}$ is finite, and so is every base change thereof. In particular, we obtain that the transform of $\mathcal{O}_{\mathcal{Z}}$ is Cohen-Macaulay, as we wanted. 
Similarly, one obtains an analog for local systems.

Theorem 5.6. We denote by $\mathcal{M}^{[n]}$ the moduli space of parabolic local systems associated to the weighted curve $\widehat{[E / \Gamma]}$ studied in Theorem 5.2. Under the assumptions of Lemma 5.4, there is a canonical equivalence of derived categories

$$
D_{\mathrm{qcoh}}\left(\mathcal{M}^{[n]}\right) \cong D_{\mathrm{qcoh}}\left(\left[[E / \Gamma]^{n} / S_{n}\right], D_{\left[[E / \Gamma]^{n} / S_{n}\right]}\right)
$$

\subsection{Moduli of parabolic local systems in positive characteristic}

In a previous paper [24], the author investigated a general relation between the moduli stacks and spaces of local systems and Higgs bundles on a curve $X$ defined over an algebraically closed field $k$ of positive characteristic. Extending a result from Bezrukavnikov-Braverman [9], it is shown there that the two moduli stacks are étale locally equivalent over the Hitchin base. The Hitchin map for local systems exists only in positive characteristic and is constructed using the $p$-curvature [33].

Let $E$ be an elliptic curve, the moduli space of rank 1 and degree 0 Higgs bundles on $E$ is given by $T^{*} E^{\vee}$; the moduli space of local systems $\mathcal{M}_{\mathrm{dR}}$ is an extension of $E^{\vee}$ by the vector space $\mathcal{A}=H^{0}\left(E, \Omega_{E}^{1}\right)$. The Hitchin map for local systems is a map

$$
\chi_{\mathrm{dR}}: \mathcal{M}_{\mathrm{dR}} \rightarrow \mathcal{A}^{(1)},
$$

where $\mathcal{A}^{(1)}$ denotes the Frobenius twist of the variety $\mathcal{A}$. Let $\omega \in H^{0}\left(E, \Omega_{E}^{1}\right)$; [30, formula 2.1.16] asserts that

$$
\tau:=\chi_{\mathrm{dR}}\left(\mathcal{O}_{E}, d+\omega\right): \mathcal{A} \rightarrow \mathcal{A}^{(1)}
$$

is a sum of a $p$-linear and a linear map. Since $\mathcal{A}$ is a $1 \mathrm{D}$ vector space, we may assume without loss of generality that this morphism is given by the Artin-Schreier morphism

$$
\text { AS }: \mathbb{A}^{1} \rightarrow \mathbb{A}^{1}
$$

which sends $\lambda \rightarrow \lambda^{p}-\lambda$. In particular, this morphism is étale. This is an explicit construction of an étale local section of the Hitchin morphism $\mathcal{M}_{\mathrm{dR}} \rightarrow \mathcal{A}^{(1)}$, that is, étale locally around every $a \in \mathcal{A}^{(1)}$ we assign a solution to the equation $a=\chi_{\mathrm{dR}}(\mathcal{O}, d+\omega)$. Any other local system $(\mathcal{E}, \nabla)$ over $a$ can now be tensored with $(\mathcal{O}, d+\omega)^{\vee}$ to obtain $\left(\mathcal{E}, \nabla^{\prime}\right)$, which is a flat connection of $p$-curvature zero. According to a theorem of Cartier, such local systems are in bijection with line bundles on the Frobenius twist $E^{(1)}$ [31, 
Theorem 5.1.1]. We conclude that after base change along $\tau$, we obtain a natural isomorphism (The author thanks C. Pauly for explaining this example to him.)

$$
\mathcal{M}_{\mathrm{dR}} \times{ }_{\mathcal{A}^{(1)}} \mathcal{A} \cong\left(E^{(1), \vee} \times \mathcal{A}^{(1)}\right) \times{ }_{\mathcal{A}^{(1)}} \mathcal{A} .
$$

One would expect that this étale local equivalence induces a similar comparison result for $\Gamma$-Hilbert schemes of the cotangent bundle of $E^{(1)}$ and $\mathcal{M}_{\mathrm{dR}}(E)$, respectively. Theorems 4.1 and 5.1 suggest that a similar étale local equivalence holds as well for certain moduli spaces of parabolic local systems. In paper [41], Nevins establishes this local equivalence on the locus of regular spectral curves (in the mirabolic case) in order to generalize Bezrukavnikov-Braverman's work on the Geometric Langlands correspondence in positive characteristic to the mirabolic case.

Strictly speaking, the parabolic case is not covered by the authors paper [24], as we assume there that $X$ is a curve. Nonetheless, the same methods used there to deduce the étale local equivalence of local systems and Higgs bundles, apply to orbicurves as well. The proof of the following proposition can also be found in the work of Poma [45].

Proposition 5.7. Let $X$ be a smooth complete orbicurve defined over an algebraically closed field $k$ of characteristic $p$, satisfying Assumption 2.13. Then the moduli stack of rank $n$ local systems $\mathcal{M}_{\mathrm{dR}}(X, n)$ is étale locally equivalent to $\mathcal{M}_{\mathrm{Dol}}\left(X^{(1)}, n\right)$ relative to the Hitchin base $\mathcal{A}^{(1)}$. The same assertion holds for (semi)stable local systems and Higgs bundles.

Proof. The only part of the proof that is sensitive to orbifold structures is [24, Theorem 3.4]. But as before it suffices to show that every $\mathbb{G}_{m}$-gerbe neutralizes on a smooth complete orbicurve $Y$ defined over an algebraically closed field. After this is established, one can evoke the same argument as in [24] to deduce representability of the stack of splittings; the corresponding result for the Picard stack is proved in [1, Theorem 5.1]. As in the curve case, one expects to be able to deduce this from Tsen's Theorem [37, Ex. III.2.22(d)], which states that for a smooth curve defined over an algebraically closed field $k$, every $\mathbb{G}_{m}$-gerbe neutralizes over the generic point

$$
H_{\mathrm{et}}^{2}\left(\operatorname{Spec} K(X), \mathbb{G}_{m}\right) .
$$

It turns out that the argument of [37] applies to our situation, after some small modifications. Let $U \subset X$ be a maximal schematic open subset of $X$; we have $K(X)=K(U)$ 
due to the birational nature of $K(X)$. We denote by $\mathcal{K}_{X}^{\times}$the sheaf of nonvanishing rational function on $X$; the sheaf-theoretic quotient $\mathcal{K}_{X}^{\times} / \mathcal{O}_{X}^{\times}$is the sheaf of $\operatorname{divisors}^{\mathrm{Div}}{ }_{X}$. By definition, we have a short exact sequence

$$
1 \rightarrow \mathcal{O}_{X}^{\times} \rightarrow \mathcal{K}_{X}^{\times} \rightarrow \operatorname{Div}_{X} \rightarrow 1
$$

called Weil divisor sequence. Taking global sections, we obtain the following interesting bit of the associated long exact sequence

$$
H_{\mathrm{et}}^{1}\left(X, \operatorname{Div}_{X}\right) \rightarrow H_{\mathrm{et}}^{2}\left(X, \mathcal{O}_{X}^{\times}\right) \rightarrow H_{\mathrm{et}}^{2}\left(X, \mathcal{K}_{X}^{\times}\right)
$$

The complement $X-U$ is a union of finitely many orbifold points $p_{1}, \ldots, p_{k}$, with stabilizer group $\Gamma_{i}$. We then have

$$
\operatorname{Div}_{X}=\bigoplus_{x \in U(k)} i_{X, *} \mathbb{Z} \oplus \bigoplus_{i=1}^{k} j_{i, *} \mathbb{Z}
$$

where $i_{X}$ denotes the closed immersion Spec $k \rightarrow X$ associated to a point $x \in U$, and $j_{i}$ denotes the closed immersion

$$
B \Gamma_{i} \rightarrow X
$$

associated to an orbifold point $p_{i}$. Since closed immersions are finite, we have $R^{l} i_{X, *}=0$ and $R^{l} j_{i, *}=0$ for $l>0$. In particular, we obtain the vanishing result $H_{\mathrm{et}}^{1}\left(X, \operatorname{Div}_{X}\right)=0$.

Let $\eta: \operatorname{Spec} K(X) \rightarrow X$ denote the inclusion of the generic point. We have

$$
\mathcal{K}_{X}^{\times}=\eta_{*} \mathbb{G}_{m}
$$

and therefore have to show that $R^{l} \eta_{*} \mathbb{G}_{m}=0$ for $l>0$. As in [37] this is checked stalkwise, by identifying $\left(R^{l} \eta_{*} \mathbb{G}_{m}\right)_{\bar{x}}$ for every $x$ different from the generic point with the Galois cohomology group

$$
H^{l}\left(\operatorname{Spec} K_{X}, \mathbb{G}_{m}\right)=0,
$$

where $K_{X}$ denotes the fraction field of the Henselization of $x$ and evoking a vanishing result in Galois cohomology due to Lang.

\section{Acknowledgements}

I thank my supervisor Tamás Hausel for explaining the structure of 2D moduli spaces of parabolic Higgs bundles to me, for introducing me to Boalch's conjecture, 
and for his remarks on this paper. Moreover, I thank Philip Boalch and Carlos Simpson for their interest in this work and for useful comments. I thank Tim Adamo and Tom Sutherland for their comments on a draft of this paper. I also thank the referee for pointing out references and suggesting how to improve the presentation of the article.

\section{Funding}

This work has been funded by an EPSRC grant received under the contract EP/G027110/1 (to M.G.).

\section{References}

[1] Aoki, M. “Hom stacks." Manuscripta Mathamatica 119, no. 1 (2006): 37-56.

[2] Arinkin, D. "Duality for representations of 1-motives." Appendix to [Donagi, R. and T. Pantev. "Torus fibrations, gerbes, and duality. With an appendix by Dmitry Arinkin." Memoirs of the American Mathematical Society 193, no. 901 (2008)], 2008.

[3] Arinkin, D. "Autoduality of compactified Jacobians for curves with plane singularities." Journal of Algebraic Geometry 22, no. 2 (2013): 363-88.

[4] Arinkin, D. and R. Bezrukavnikov. "Perverse coherent sheaves." Moscow Mathematical Journal 10, no. 1 (2010): 3-29.

[5] Artin, M. "Versal deformations and algebraic stacks." Inventiones Mathematicae 27, no. 3 (1974): 165-89.

[6] Atiyah, M. F. "Complex analytic connections in fibre bundles." Transactions of the American Mathematical Society 85 (1957): 181-207.

[7] Beauville, A., M. S. Narasimhan, and S. Ramanan. "Spectral curves and the generalised theta divisor." Journal für die Reine und Angewandte Mathematik 398 (1989): 169-79.

[8] Ben-Zvi, D., J. Francis, and D. Nadler. "Integral transforms and Drinfeld centers in derived algebraic geometry." Journal of the American Mathematical Society 23, no. 4 (2010): 909-66.

[9] Bezrukavnikov, R. and A. Braverman. "Geometric Langlands correspondence for D-modules in prime characteristic: the GL(n) case." Pure and Applied Mathematics. (2007), no. 1, Special Issue: In honor of Robert D. MacPherson. Part 3, 153-79.

[10] Bezrukavnikov, R., I. Mirković, and D. Rumynin. "Localization of modules for a semisimple Lie algebra in prime characteristic." Annals of Mathematics (2) 167, no. 3 (2008): 945-991.

[11] Biquard, O. and P. Boalch. "Wild non-abelian Hodge theory on curves." Compositio Mathematica 140, no. 1 (2004): 179-204.

[12] Biswas, I. and V. Heu. "On the logarithmic connections over curves." (2012): preprint http://arxiv.org/abs/1205.2676.

[13] Boalch, P. "Simply-laced isomonodromy systems." (2011): preprint http://arxiv.org/abs/ $1107.0874 \mathrm{v} 1$.

[14] Boden, H. U. and K. Yokogawa. "Moduli spaces of parabolic Higgs bundles and parabolic $K(D)$ pairs over smooth curves. I." International Journal of Mathematics 7, no. 5 (1996): 573-98. 
[15] Borne, N. "Fibrés paraboliques et champ des racines." International Mathematics Research Notices, no. 16 (2007).

[16] Briançon, J. “Description de $H \mathrm{ilb}^{n} C\{x, y\}$." Inventiones Mathematicae 41, no. 1 (1977): $45-89$.

[17] Bridgeland, T. and S. Iyengar. "A criterion for regularity of local rings." Comptes Rendus Mathématique. Académie des Sciences. Paris 342, no. 10 (2006): 723-26.

[18] Bridgeland, T., A. King, and M. Reid. "Mukai implies McKay: the McKay correspondence as an equivalence of derived categories." Journal of the American Mathematical Society 14, no. 3 (2001): 535-54.

[19] Chen, T.-H. and X. Zhu. "Geometric Langlands in prime characteristic." (PhD diss., Massachusetts Institute of Technology, 2012).

[20] Donagi, R. and T. Pantev. "Langlands duality for Hitchin systems." Inventiones Mathematicae 189, no. 3 (2012): 653-735.

[21] Fantechi, B., L. Göttsche, L. Illusie, S. L. Kleiman, N. Nitsure, and A. Vistoli. Fundamental Algebraic Geometry. Mathematical Surveys and Monographs 123 (American Mathematical Society, Providence, RI, 2005). Grothendieck's FGA explained.

[22] Furuta, M. and B. Steer. "Seifert fibred homology 3-spheres and the Yang-Mills equations on Riemann surfaces with marked points." Advances in Mathematics, 96, no. 1 (1992): 38-102.

[23] Gorsky, A., N. Nekrasov, and V. Rubtsov. "Hilbert schemes, separated variables, and Dbranes." Communications in Mathematical Physics 222, no. 2 (2001): 299-318.

[24] Groechenig, M. "Moduli of flat connections in positive characteristic." (2012): preprint http://arxiv.org/abs/1201.0741.

[25] Haiman, M. "Hilbert schemes, polygraphs and the Macdonald positivity conjecture." Journal of the American Mathematical Society 14, no. 4 (2001): 941-1006 (electronic).

[26] Hausel, T., E. Letellier, and F. Rodriguez-Villegas. "Arithmetic harmonic analysis on character and quiver varieties." Duke Mathematical Journal 160, no. 2 (2011): 323-400.

[27] Hitchin, N. "Stable bundles and integrable systems." Duke Mathematical Journal 54, no. 1 (1987): 91-114.

[28] Hitchin, N. J. "The self-duality equations on a Riemann surface." Proceedings of the London Mathematical Society. Third Series 55, no. 1 (1987): 59-126.

[29] Iarrobino, A. "Punctual Hilbert schemes." American Mathematical Society. Bulletin 78, no. 5 (1972): 819-23.

[30] Illusie, L. "Complexe de de Rham-Witt et cohomologie cristalline." Annales Scientifiques de l'École Normale Supérieure. Quatriéme Série 12, no. 4 (1979): 501-661.

[31] Katz, N. M. "Nilpotent connections and the monodromy theorem: applications of a result of turrittin." Publications Mathématiques. Institut de Hautes Études Scientifiques 39, no. 1 (1970): 175-232.

[32] Kostov, V. P. "The Deligne-Simpson problem for zero index of rigidity." Perspectives of Complex Analysis, Differential Geometry and Mathematical Physics (St. Konstantin, 2000), no. 1 (2001): 1-35. River Edge, NJ:World Scientific Publisher.

[33] Laszlo, Y. and C. Pauly. “On the Hitchin morphism in positive characteristic." International Mathematics Research Notices 2001, no. 3 (2001): 129-43. 
[34] Laumon, G. “Transformation de Fourier généralisée." (1996): preprint http://arxiv.org/ abs/alg-geom/9603004.

[35] Laumon, G. and L. Moret-Bailly. Champs algébriques. Ergebnisse der Mathematik und ihrer Grenzgebiete. 3. Folge. A Series of Modern Surveys in Mathematics [Results in Mathematics and Related Areas. 3rd Series. A Series of Modern Surveys in Mathematics] 39. Berlin: Springer, 2000.

[36] Lurie, J. “Stable infinity categories." (2006): preprint http://arxiv.org/abs/math/0608228.

[37] Milne, J. S. Étale Cohomology. Princeton Mathematical Series 33 (Princeton University Press, Princeton, NJ, 1980).

[38] Mukai, S. "Fourier Functor and its Application to the Moduli of Bundles on an Abelian Variety." Algebraic Geometry, Sendai, 1985, 515-50. Advanced Studies in Pure Mathematics 10. Amsterdam: North-Holland, 1987.

[39] Nakajima, H. "Hyper-Kähler Structures on Moduli Spaces of Parabolic Higgs Bundles on Riemann Surfaces." Moduli of Vector Bundles (Sanda, 1994; Kyoto, 1994), 199-208. Lecture Notes in Pure and Applied Mathematics 179. New York: Dekker, 1996.

[40] Nasatyr, B. and B. Steer. "Orbifold Riemann surfaces and the Yang-Mills-Higgs equations." Annali della Scuola Normale Superiore di Pisa. Classe di Scienze. Serie IV 22, no. 4 (1995): 595-643.

[41] Nevins, T. "Mirabolic Langlands duality and the quantum Calogero-Moser system." Transformation Groups 14, no. 4 (2009): 931-83.

[42] Nitsure, N. "Moduli space of semistable pairs on a curve." Proceedings of the London Mathematical Society. Third Series 62, no. 2 (1991): 275-300.

[43] Okamoto, K. "Studies on the Painlevé equations. I. Sixth Painlevé equation PV." Annali di Matematica Pura ed Applicata. Series IV 146 (1987): 337-81.

[44] Ploog, D. "Equivariant autoequivalences for finite group actions." Advances in Mathematics (1) 216: 62-74.

[45] Poma, F. "Étale cohomology of a DM curve-stack with coefficients in $\mathbb{G}_{m}$. " Monatshefte für Mathematik 169, no. 1 (2013): 33-50.

[46] Reineke, M. "Moduli of Representations of Quivers." Trends in Representation Theory of Algebras and Related Topics, EMS Series of Congress Reports, 589-637. Zürich: European Mathematical Society, 2008.

[47] Roberts, P. “Intersection theorems." Commutative Algebra, 417-436. MSRI Publications 15. Berlin: Springer, 1989.

[48] Rothstein, M. "Sheaves with connection on abelian varieties." Duke Mathematical Journal 84, no. 3 (1996): 565-98.

[49] Serre, J.-P. Algebraic Groups and Class Fields. Graduate Texts in Mathematics 117. New York: Springer, 1988. Translated from the French.

[50] Silverman, J. H. Advanced Topics in the Arithmetic of Elliptic Curves. Graduate Texts in Mathematics 151. New York: Springer, 1994.

[51] Simpson, C. T. "Harmonic bundles on noncompact curves." Journal of the American Mathematical Society 3, no. 3 (1990): 713-70. 
[52] Simpson, C. T. "Higgs bundles and local systems." Publications Mathématiques. Institut de Hautes Études Scientifiques 75 (1992): 5-95.

[53] Simpson, C. T. "Moduli of representations of the fundamental group of a smooth projective variety. II." Publications Mathématiques. Institut de Hautes Études Scientifiques 80, no. 1 (1994): 5-79.

[54] Sosna, P. "Linearisations of triangulated categories with respect to finite group actions." Mathematical Research Letters 19, no. 5 (2012) 1007-20.

[55] The Stacks Project Authors. Stacks project. http://math.columbia.edu/algebraic_geometry/ stacks-git.

[56] Toen, B. “Théorèmes de Riemann-Roch pour les champs de Deligne-Mumford." K-Theory, 18, no. 1 (1999): 33-76.

[57] Yokogawa, K. "Compactification of moduli of parabolic sheaves and moduli of parabolic Higgs sheaves." Journal of Mathematics of Kyoto University 33, no. 2 (1993): 451-504. 